JOURNAL OF THE

AMERICAN MATHEMATICAL SOCIETY

Volume 10, Number 4, October 1997, Pages 835-864

S 0894-0347(97)00241-5

\title{
DEHN SURGERY ON KNOTS IN 3-MANIFOLDS
}

\author{
MARC LACKENBY
}

\section{INTRODUCTION}

It has been known for over 30 years that every closed connected orientable 3manifold is obtained by surgery on a link in $S^{3}$ [8]. However, a classification of such 3-manifolds in terms of this surgery construction has remained elusive. This is due primarily to the lack of uniqueness of the surgery description. In [5], Kirby gave us a calculus of surgery diagrams. However, the lack of a 'canonical' surgical method of getting from one 3-manifold to another has hampered further progress. In this paper, we show that if one restricts attention to the case where a surgery curve is homotopically trivial in a 3-manifold, then one has the following uniqueness theorem.

Theorem 4.1. For $i=1$ and 2 , let $V^{i}$ be a compact connected oriented 3-manifold, possibly with boundary, such that $H_{1}\left(V^{i} ; \mathbb{Q}\right) \neq 0$. Let $K_{i}$ be a homotopically trivial knot in $V^{i}$, such that $V^{i}-K_{i}$ is irreducible and atoroidal. Let $V_{K_{i}}^{i}\left(p_{i} / q_{i}\right)$ be the manifold obtained from $V^{i}$ by $p_{i} / q_{i}$ Dehn surgery along $K_{i}$, where $p_{i}$ and $q_{i}$ are coprime integers. Then, there is a natural number $C\left(V^{1}, K_{1}\right)$, which depends only on $V^{1}$ and $K_{1}$, with the following property. If $\left|q_{2}\right|>C\left(V^{1}, K_{1}\right)$, then

$$
V_{K_{1}}^{1}\left(p_{1} / q_{1}\right) \cong V_{K_{2}}^{2}\left(p_{2} / q_{2}\right) \Longleftrightarrow\left\{\begin{array}{c}
\left(V^{1}, K_{1}\right) \cong\left(V^{2}, K_{2}\right) \\
\text { and } \\
p_{1} / q_{1}=p_{2} / q_{2}
\end{array}\right\},
$$

where $\cong$ denotes an orientation-preserving homeomorphism.

The above result admits several generalisations. For example, there is a version which deals with orientation-reversing homeomorphisms. (See Remark 4.8.) There are also many examples which demonstrate that some restriction on $q_{2}$ is necessary in Theorem 4.1. (See Example 4.9.)

Theorem 4.1 demonstrates the sheer plethora of 3-manifolds. For if $V^{1}$ and $V^{2}$ are distinct oriented 3-manifolds, or $K_{1}$ and $K_{2}$ are distinct knots (up to orientationpreserving homeomorphism), or $p_{1} / q_{1} \neq p_{2} / q_{2}$, then $V_{K_{1}}^{1}\left(p_{1} / q_{1}\right)$ and $V_{K_{2}}^{2}\left(p_{2} / q_{2}\right)$ are distinct, if $\left|q_{2}\right|$ is sufficiently large. Thus, $V_{K_{1}}^{1}\left(p_{1} / q_{1}\right)$ 'encodes' $V^{1}, K_{1}$ and $p_{1} / q_{1}$, if $\left|q_{1}\right|>C\left(V^{1}, K_{1}\right)$. It is interesting to note how this is done. It is trivial that the homology of $V_{K_{1}}^{1}\left(p_{1} / q_{1}\right)$ and $V^{1}$ determines $\left|p_{1}\right|$. But $V_{K_{1}}^{1}\left(p_{1} / q_{1}\right)$ 'stores' $\left|q_{1}\right|$ as follows. If $M$ is a compact connected orientable irreducible 3-manifold, possibly with boundary, which is neither a rational homology 3-sphere nor a 3-ball, we define an integer-valued homeomorphism invariant $I(M)$ of $M$. It has the following property.

Received by the editors April 26, 1996 and, in revised form, April 22, 1997.

1991 Mathematics Subject Classification. Primary 57N10; Secondary 57M25.

(C)1997 American Mathematical Society 
Theorem 4.2. Let $V^{1}$ and $K_{1}$ be as in Theorem 4.1. If $\left|q_{1}\right| \geq C\left(V^{1}, K_{1}\right)$, then $V_{K_{1}}^{1}\left(p_{1} / q_{1}\right)$ is irreducible, and $I\left(V_{K_{1}}^{1}\left(p_{1} / q_{1}\right)\right)=\left|q_{1}\right|$.

The invariant $I(M)$ encodes other very relevant surgery information. It arises naturally from consideration of the following problem. When does Dehn surgery on a knot $L$ in $M$ yield a reducible 3-manifold? A useful approach to this problem has been to study the integer $\Delta(r, \mu)$, where $\mu$ is the meridional slope on the boundary of a regular neighbourhood $\mathcal{N}(L)$ of $L, r$ is the surgery slope and $\Delta(-,-)$ is the standard notion of distance between slopes on the torus. For example, it was proved in [2] that, when $M$ is $S^{3}$, then $\Delta(r, \mu)=1$. It has been generally believed, though, that it is impossible to say very much for arbitrary $M$. The following theorem demonstrates very clearly that this belief is mistaken.

Theorem 2.9. Let $M$ be a compact connected orientable irreducible 3-manifold, possibly with boundary. Let $L$ be a knot in $M$ which does not lie in a 3-ball. Suppose that $H_{2}(M-\operatorname{int}(\mathcal{N}(L)), \partial M) \neq 0$. Let $\mu$ be the meridional slope on $\partial \mathcal{N}(L)$. Let $M_{L}(r)$ be the manifold obtained from $M$ by Dehn surgery along $L$ with slope $r$. Suppose that at least one of the following holds.

(a) There is a sphere in $M_{L}(r)$ which does not bound a rational homology 3-ball, or

(b) there is no essential torus in $M$ which bounds a rational homology solid torus, and $M_{L}(r)$ is reducible, or

(c) the core of the surgery solid torus in $M_{L}(r)$ has finite order in $\pi_{1}\left(M_{L}(r)\right)$.

Then there is an upper bound on $\Delta(r, \mu)$ which depends only on $M$.

In fact, the upper bound in Part (b) is $I(M)$. Now, the invariant $I(M)$ is, in general, difficult to calculate. However, an upper bound on $I(M)$ is relatively easy to obtain. First, one finds a taut sutured manifold structure $(M, \gamma)$ on $M$ (using the language of [9]), which always exists by Theorem 2.1. Next, one constructs a sutured manifold hierarchy for $(M, \gamma)$ and then one performs a few easy computations. Moreover, for the purposes of Theorem 2.9, an upper bound on $I(M)$ is all that we require. For example, we prove the following theorem by showing that $I(M) \leq 1$.

Theorem 3.2. Let $M$ be $F \times S^{1}$, where $F$ is a compact connected orientable surface, possibly with boundary, which is neither a sphere nor a disc. Let $L$ be a knot in $M$ which does not lie in a 3-ball. Let $\mu$ be the meridional slope on $\partial \mathcal{N}(L)$. Let $M_{L}(r)$ be the manifold obtained from $M$ by Dehn surgery along $L$ with slope $r \neq \mu$. If $M_{L}(r)$ is reducible or the core of the surgery solid torus has finite order in $\pi_{1}\left(M_{L}(r)\right)$, then $\Delta(r, \mu)=1$.

Theorem 2.9 should be compared with the results of [7]. That paper deals with the case where $M$ is the exterior of an oriented link $K$ in $S^{3}$ and $L$ is a curve in $S^{3}$ bounding a disc which intersects $K$ in two points of opposite sign. Dehn surgery with coefficient \pm 1 along such a curve $L$ is the standard notion of a crossing change. When does $1 / q$ surgery along $L$ yield the unknot or a split link? In that paper, an upper bound on $|q|$ was discovered which depends only on $K$ and which can easily be read from a single diagram of $K$ of a certain type. This type includes all non-split alternating diagrams.

Thus, throughout most of this paper, the integer $\Delta(r, \mu)$ is studied, where $\mu$ is the meridional slope, $r$ is the surgery slope and $M_{L}(r)$ is a reducible manifold. Assume, for the moment, that $L$ is homologically trivial in $M$. Then there is also a longitudinal slope $\lambda$ on $\partial \mathcal{N}(L)$. What can be said about $\Delta(r, \lambda)$ ? We show that 
there is no upper bound on $\Delta(r, \lambda)$ which depends only on $M$. Nevertheless, we can still obtain interesting information. $L$ bounds an orientable surface and the genus of $L$ is defined to be the minimal genus of such a surface. In $\S 5$, we find an upper bound on $\Delta(r, \lambda)$ which depends only on the genus of $L$. In fact, we do not need the assumption that $L$ is homologically trivial. We generalise the notion of a longitudinal slope on $\partial \mathcal{N}(L)$ to incorporate arbitrary knots $L$. An upper bound on $\Delta(r, \lambda)$ is found in terms of the associated genus.

Throughout this paper, we shall use the following terminology and conventions. Manifolds may be disconnected, or even empty, where appropriate. A submanifold of a given manifold with non-zero codimension is always properly embedded and compact. If $L$ is a submanifold of a manifold $M$, we let $\mathcal{N}(L)$ be a regular neighbourhood of $L$ in $M$. If $M$ is a 3-manifold and $L$ is a $\operatorname{knot}$ in $M$, then we let $M_{L}=M-\operatorname{int}(\mathcal{N}(L))$. If $N$ is a 3-manifold with a specified toral boundary component $P$, and $r$ is a slope on $P$ (that is, the isotopy class of an essential simple closed curve on $P$ ), we let $N(r)$ be the manifold obtained by gluing in a solid torus to $P$ so that the meridional slope on the boundary of the solid torus is identified with slope $r$ on $P$. In the manifold $M_{L}, \partial \mathcal{N}(L)$ will always be the specified torus. Hence, $M_{L}(r)$ is a manifold obtained from $M$ by Dehn surgery on $L$.

The techniques of this paper are those of sutured manifold theory. We shall use the version of this theory which is developed in $\S \S 0-7$ of [9]. The approach to the proof of Theorem 2.9 is as follows. We first show (Theorem 2.1) that $M$ can be given a taut sutured manifold structure $(M, \gamma)$. Theorem 4.19 of [9] implies that there is a taut sutured manifold sequence in which the first sutured manifold is $(M, \gamma)$, and in which the final sutured manifold is a collection of 3-balls. We assume that $\Delta(r, \mu)>I(M)$. Then we use a version of the main result (Theorem 1.4) of [6] which implies that, given any orientable surface $F$ in $M$, then $L$ can be isotoped away from $F$ if $\Delta(r, \mu)$ is sufficiently large. We apply this to the decomposing surfaces of the sutured manifold sequence to deduce that $L$ can be isotoped so that it lies in a collection of balls. This is the required contradiction. The integer $I(M)$ is essentially the smallest integer for which this argument works. This is what is done in Theorem 2.5. However, to apply Theorem 1.4 of [6], there are a number of conditions on tori in $M_{L}$ which must be satisfied. To establish these, it is necessary to prove a number of technical lemmas and to provide stronger versions of well-known results in sutured manifold theory. These details are relegated to an appendix, and are referred to by the prefix A.

The proof of Theorem 4.1 is similar to that of Theorem 2.9, but more complicated. We find a taut sutured manifold sequence, the first manifold being $V_{K_{1}}^{1}\left(p_{1} / q_{1}\right)$ and the final manifold being a solid torus neighbourhood of the surgery curve $L_{1}$ together with a collection of balls. We show that if $L_{2}$ is any other suitable surgery curve in $V_{K_{1}}^{1}\left(p_{1} / q_{1}\right)$, then there is an ambient isotopy of $L_{2}$ which pulls it clear of each of the decomposing surfaces and onto $L_{1}$. This again uses Theorem 1.4 of $[6]$. Theorem 4.1 follows fairly rapidly.

\section{Producing Reducible manifolds by Dehn surgery}

The goal of this section is to prove Theorem 2.9, which was given in $\S 1$. First, we must show that we can always apply sutured manifold theory.

Theorem 2.1. Let $M$ be a compact orientable irreducible 3-manifold, possibly with boundary. Then there is a taut sutured manifold structure on $M$. 


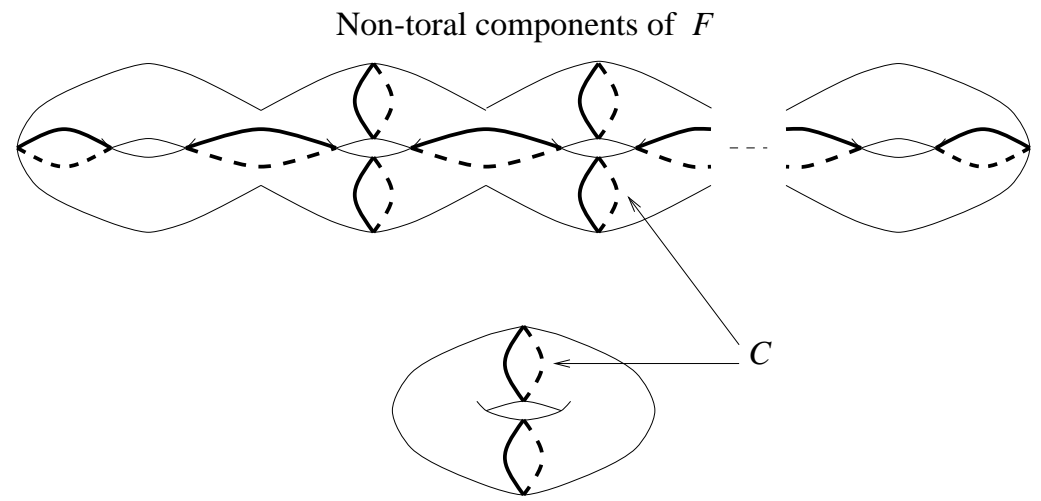

Toral components of $F$

Figure 1

Lemma 2.2. Let $M$ be a compact orientable 3-manifold with incompressible boundary. Suppose that $F$ is a surface homeomorphic to $\partial M$. Let $C$ be a union of essential disjoint simple closed curves in $F$, no two of which are parallel. Then, there exists a homeomorphism $h: F \rightarrow \partial M$ such that no two curves in $h(C)$ are co-annular in $M$.

Proof. This is essentially contained in Lemma 5.2 of [10]. The proof is by induction on the number $|C|$ of components of $C$. It is trivially true for $|C|=1$. Hence, we assume it is true for $|C|=N$. Suppose that $|C|=N+1$. Pick a curve $C^{\prime}$ in $C$. Then there exists a required homeomorphism $h$ for $C-C^{\prime}$. Suppose that $h\left(C^{\prime}\right)$ is co-annular with a curve in $h\left(C-C^{\prime}\right)$. Then, the techniques of Lemma 5.2 of [10] demonstrate how to modify $h$ to $h_{1}$ so that $h_{1}\left(C-C^{\prime}\right)=h\left(C-C^{\prime}\right)$ and so that $h_{1}\left(C^{\prime}\right)$ is not co-annular with any curve of $h_{1}\left(C-C^{\prime}\right)$. More specifically, consider the component $E$ of $F-\operatorname{int}\left(\mathcal{N}\left(C-C^{\prime}\right)\right)$ containing $C^{\prime}$. Then, the argument of Lemma 5.2 of [10] gives a homeomorphism $h_{2}: F \rightarrow F$ which is supported in $E$, such that $h_{1}=h \circ h_{2}$ is of the required form.

Proof of Theorem 2.1. Consider first the case where $\partial M$ is incompressible and contains no sphere components. Let $F$ be a surface homeomorphic to $\partial M$. Let $C$ be a union of simple closed curves in $F$, such that for each component of $F$, they are of the form shown in Figure 1.

It is clear that such a collection of curves divides $F$ into two surfaces $F_{-}$and $F_{+}$ such that $F_{-} \cap F_{+}=C$. Let $C^{\prime}$ be a subset of $C$ which contains exactly one curve on each toral component of $F$. Then no two curves of $C-C^{\prime}$ are co-annular in $F$. By Lemma 2.2, there is a homeomorphism $h: F \rightarrow \partial M$ such that no two curves in $h\left(C-C^{\prime}\right)$ are co-annular in $M$. Hence, two curves in $h(C)$ are co-annular in $M$ if and only if they are co-annular in $\partial M$. We place a sutured manifold structure on $M$, by letting $R_{ \pm}=h\left(F_{ \pm}\right)$and letting $\gamma=h(C)$. We shall show that it is taut.

This is essentially contained in the proof of Proposition 5.8 (a) of [10]. Note that $\chi_{-}\left(R_{-}\right)=\chi_{-}\left(R_{+}\right)=-\chi(\partial M) / 2$. Let $\mathcal{T}$ be the set of toral components of $\partial M$. Then, for each component $X$ of $\partial M$,

$$
|\gamma \cap X|= \begin{cases}-3 \chi(X) / 2, & \text { if } X \notin \mathcal{T} ; \\ 2, & \text { if } X \in \mathcal{T} .\end{cases}
$$


Hence, $|\gamma|=-3 \chi(\partial M) / 2+2|\mathcal{T}|$. Let $S$ be a compact oriented surface in $M$ with $\partial S=\gamma$. Since $\partial M$ is incompressible, no component is a disc. There are at most $|\mathcal{T}|$ annuli. If $S^{\prime}$ is a component of $S$ with genus $\left(S^{\prime}\right) \geq 1$, then $\chi_{-}\left(S^{\prime}\right)=-\chi\left(S^{\prime}\right)=$ $2\left(\operatorname{genus}\left(S^{\prime}\right)\right)-2+\left|\partial S^{\prime}\right| \geq\left|\partial S^{\prime}\right|$. If genus $\left(S^{\prime}\right)=0$ and $S^{\prime}$ is neither an annulus nor a sphere, then $\chi_{-}\left(S^{\prime}\right)=-2+\left|\partial S^{\prime}\right| \geq\left|\partial S^{\prime}\right| / 3$. Thus, $\chi_{-}(S) \geq(|\partial S|-2|\mathcal{T}|) / 3=$ $-\chi(\partial M) / 2=\chi_{-}\left(R_{ \pm}\right)$. Hence, $R_{ \pm}$is taut in $M$. This proves the theorem when $M$ has incompressible boundary and $\partial M$ has no sphere components. Note that the above argument could have been simplified if we had instead placed no sutures on toral components of $\partial M$. However, in what follows, it is helpful to have each component of $\partial M$ containing at least one suture.

If $\partial M$ has any sphere components, these bound balls in $M$. We may place a taut sutured manifold structure on these balls, so that each ball contains a single suture.

Suppose now that $\partial M$ is compressible. Let $M_{1}$ be a 3 -manifold obtained by boundary-reducing $M$ as far as possible. Then $M_{1}$ is an irreducible manifold with incompressible boundary, and so has a taut sutured manifold structure $\left(M_{1}, \gamma_{1}\right)$ by the above argument. Moreover, each component of $\partial M_{1}$ contains at least one suture. $M$ is obtained, up to homeomorphism, from $M_{1}$ by identifying in pairs a number of disjoint discs in $\partial M_{1}$. We may isotope these discs without changing the homeomorphism type of $M$. We may assume that each disc intersects $\gamma_{1}$ in a single arc on the boundary of the disc. We also ensure that, if two discs are identified, then one lies in $R_{-}$and one in $R_{+}$. Thus, there is a sutured manifold structure $(M, \gamma)$ and a collection of product discs in $M$ so that decomposing along these discs yields $\left(M_{1}, \gamma_{1}\right)$. By Theorem 3.6 of [9], $(M, \gamma)$ is taut.

We now introduce the 3-manifold invariant which is central to this paper.

Definition 2.3. Let $M_{1}$ be a compact connected orientable irreducible 3-manifold, possibly with boundary. Let $\left(M_{1}, \gamma_{1}\right)$ be a taut sutured manifold structure, which exists by Theorem 2.1. Suppose that $H_{2}\left(M_{1}, \partial M_{1}\right)$ is non-trivial and that $z_{\mu}$ is an element of $H_{2}\left(M_{1}, \partial M_{1}\right)$. Let $\mathcal{H}$ be a taut sutured manifold sequence

$$
\left(M_{1}, \gamma_{1}\right) \stackrel{S_{1}}{\longrightarrow}\left(M_{2}, \gamma_{2}\right) \stackrel{S_{2}}{\longrightarrow} \ldots \stackrel{S_{n-1}}{\longrightarrow}\left(M_{n}, \gamma_{n}\right),
$$

such that $M_{n}$ is a collection of balls, and such that no component of $\partial S_{i}$ bounds a disc in $R_{ \pm}\left(\gamma_{i}\right)$, for any $i$. Ensure that $\left[S_{1}, \partial S_{1}\right]=z_{\mu} \in H_{2}\left(M_{1}, \partial M_{1}\right)$. If $z_{\mu} \neq$ 0 , such a sequence exists by Theorems 2.6 and 4.19 of [9]. Let $\mathcal{S}$ be the set of components of $\bigcup_{i=1}^{n} M_{i}$ which are solid tori. Let $w(\mathcal{H})$ be the maximum winding number of any single suture in an element of $\mathcal{S}$. Let $w(\mathcal{H})=0$ if $\mathcal{S}=\emptyset$. Let $\left\{S_{i}^{j}: j \in \mathcal{C}_{i}\right\}$ be the set of components of $S_{i}$ which are not in an element of $\mathcal{S}$. Let

$$
I(\mathcal{H})=\max \left\{\max _{1 \leq i \leq n-1} \max _{j \in \mathcal{C}_{i}}\left\{-\chi\left(S_{i}^{j}\right)+\frac{\left|S_{i}^{j} \cap \gamma_{i}\right|}{2}+1\right\}, w(\mathcal{H})\right\} .
$$

In the above, we follow the convention that the maximum value of an empty set of integers is zero. Define $I\left(M_{1}, \gamma_{1}, z_{\mu}\right)$ to be the minimum of $I(\mathcal{H})$ over all such sequences $\mathcal{H}$. If $z_{\mu}=0$ and there is no such sequence $\mathcal{H}$, define $I\left(M_{1}, \gamma_{1}, z_{\mu}\right)$ to be infinite. Define $I\left(M_{1}, \gamma_{1}\right)$ to be the minimum of $I\left(M_{1}, \gamma_{1}, z_{\mu}\right)$ over all $z_{\mu}$. Define $I\left(M_{1}\right)$ to be the minimum of $I\left(M_{1}, \gamma_{1}\right)$ over all possible taut sutured manifold structures on $M_{1}$.

Since the invariant $I\left(M_{1}\right)$ is defined to be the minimum of an infinite collection, it is in general difficult to calculate. Nevertheless, an upper bound on $I\left(M_{1}\right)$ is 
often all that is required, and this is relatively straightforward to obtain. First find a taut sutured manifold structure $\left(M_{1}, \gamma_{1}\right)$, which always exists by Theorem 2.1. Then find a suitable taut sutured manifold sequence $\mathcal{H}$ (for example, a hierarchy) for $\left(M_{1}, \gamma_{1}\right)$, and calculate $I(\mathcal{H})$. An example is given in $\S 3$.

Lemma 2.4. Let $\left(M_{1}, \gamma_{1}\right)$ be a connected taut sutured manifold. Let $z_{\mu}$ be a nontrivial element of $H_{2}\left(M_{1}, \partial M_{1}\right)$. Then $I\left(M_{1}\right), I\left(M_{1}, \gamma_{1}\right)$ and $I\left(M_{1}, \gamma_{1}, z_{\mu}\right)$ are positive integers.

Proof. Pick a sequence $\mathcal{H}$ as in Definition 2.3. For each $i, \gamma_{i}$ divides $\partial M_{i}$ into $R_{ \pm}\left(\gamma_{i}\right)$, and hence $\left|S_{i}^{j} \cap \gamma_{i}\right|$ is even. This implies that $I\left(M_{1}\right), I\left(M_{1}, \gamma_{1}\right)$ and $I\left(M_{1}, \gamma_{1}, z_{\mu}\right)$ are integers.

Suppose that $I(\mathcal{H})$ is not positive. Then each surface $S_{i}^{j}$ must be a sphere or disc disjoint from $\gamma_{i}$ and $w(\mathcal{H})$ must be zero. However, if $\mathcal{S} \neq \emptyset$, then $w(\mathcal{H})>0$, since each sutured manifold in $\mathcal{H}$ is taut. Hence, $\mathcal{S}=\emptyset$. Therefore each component of $S_{i}$ belongs to $\mathcal{C}_{i}$. No $S_{i}^{j}$ is a 2 -sphere, since $M_{i+1}$ is irreducible and $M_{i}$ is not $S^{3}$. If $S_{i}^{j}$ is a disc disjoint from $\gamma_{i}$, then $\partial S_{i}^{j}$ bounds a disc in $R_{ \pm}\left(\gamma_{i}\right)$, since $\left(M_{i}, \gamma_{i}\right)$ is taut. This is a contradiction.

The following theorem is the first and most important step in proving Theorem 2.9. It demonstrates the usefulness of the invariant $I(M)$. Recall the well-known definition of a rational homology cobordism (Definition A.3).

Theorem 2.5. Let $(M, \gamma)$ be a connected taut sutured manifold. Let $L$ be a knot in $M$ which does not lie in a 3-ball. Suppose that $H_{2}\left(M_{L}, \partial M\right)$ is non-trivial. Let $\mu$ be the meridional slope on $\partial \mathcal{N}(L)$ and let $r$ be another slope. Suppose that the only tori in $M_{L}$ which are cobordant to $\partial \mathcal{N}(L)$ by a rational homology cobordism are parallel to $\partial \mathcal{N}(L)$.

(a) If $\left(M_{L}(r), \gamma\right)$ is not taut, then $\Delta(r, \mu) \leq I(M, \gamma)$. In particular, if $M_{L}(r)$ is reducible, then $\Delta(r, \mu) \leq I(M)$.

(b) If the core of the surgery solid torus in $M_{L}(r)$ has finite order in $\pi_{1}\left(M_{L}(r)\right)$, then $\Delta(r, \mu) \leq I(M)$.

(c) Suppose that $\partial M$ is a (possibly empty) collection of tori and that $\gamma=\emptyset$. Let $z$ be a non-trivial element of $H_{2}\left(M_{L}, \partial M\right)$. Let $z_{\mu}$ and $z_{r}$ be the elements of $H_{2}(M, \partial M)$ and $H_{2}\left(M_{L}(r), \partial M_{L}(r)\right)$, respectively, to which $z$ is sent by the maps induced by inclusion. If the Thurston norm of $z_{r}$ is less than that of $z_{\mu}$ or $z$, then $\Delta(r, \mu) \leq I\left(M, \gamma, z_{\mu}\right)$.

Note that $I(M), I(M, \gamma)$ and $I\left(M, \gamma, z_{\mu}\right)$ do not depend on $L$. The following lemma is immediate.

Lemma 2.6. Suppose that $(M, \gamma)$ is a connected taut sutured manifold. Let $L$ be a knot in $M$ which does not lie in a 3-ball. Suppose that $M_{L}$ is not a solid torus. Then the sutured manifold $\left(M_{L}, \gamma\right)$, where $R_{+}\left(M_{L}\right)=R_{+}(M)$ and $R_{-}\left(M_{L}\right)=$ $R_{-}(M) \cup \partial \mathcal{N}(L)$, is taut.

Proof of Theorem 2.5. In Part (b), we may assume that $I(M)=I(M, \gamma)$. Suppose that the inequality of the theorem does not hold. That is, assume, in Parts (a) and (b), that $\Delta(r, \mu)>I(M, \gamma)$, and, in Part (c), that $\Delta(r, \mu)>I\left(M, \gamma, z_{\mu}\right)$. Note that, by Lemma 2.4 , this implies that $\Delta(r, \mu)>1$. Let $\left(M_{1}, \gamma_{1}\right)=(M, \gamma)$. Let $\mathcal{H}$ be a sutured manifold sequence as in Definition 2.3. In Parts (a) and (b), we insist that $I(\mathcal{H})=I(M, \gamma)$. In Part (c), we insist that the first decomposing surface $S_{1}$ 
of $\mathcal{H}$ satisfies $\left[S_{1}, \partial S_{1}\right]=z_{\mu} \in H_{2}(M, \partial M)$, and that $I(\mathcal{H})=I\left(M, \gamma, z_{\mu}\right)$. Hence, in Parts (a), (b) and (c), $\Delta(r, \mu)>I(\mathcal{H})$.

We shall show, by induction on $i$, that there is an ambient isotopy of $L$ in $M$, so that, after the isotopy, $L$ lies in $M_{i}$ for each $i$. This will provide a contradiction. For, $L$ does not lie in a 3-ball, but $M_{n}$ is a collection of 3-balls.

The induction hypothesis is trivially true for $i=1$. Suppose it is true for a given $i$. By Lemma 2.6, the sutured manifold $\left(M_{i}-\operatorname{int}(\mathcal{N}(L)), \gamma_{i}\right)$ is taut. We wish to use Theorem A.10 to deduce that there is an ambient isotopy of $L$ in $M_{i}$, so that, after the isotopy, $L$ is disjoint from $S_{i}$, and so $L$ may be viewed as lying in $M_{i+1}$. Let $M_{i}^{\prime}$ be the manifold obtained from $M_{i}$ by Dehn surgery along $L$ with slope $r$. Let $L^{\prime}$ be the core of the surgery solid torus in $M_{i}^{\prime}$. Let $V$ be the component of $M_{i}$ containing $L$.

Claim. In Part (a), $\left(M_{i}^{\prime}, \gamma_{i}\right)$ is not taut. In Part (b), either $\left(M_{i}^{\prime}, \gamma_{i}\right)$ is not taut or $\left[L^{\prime}\right]$ has finite order in $\pi_{1}\left(V_{L}(r)\right)$, or both. In Part (c), $\left(M_{i}^{\prime}, \gamma_{i}\right)$ is not taut, if $i \geq 2$.

Now, by assumption, no component of $\partial S_{j}$ bounds a disc in $R_{ \pm}\left(\gamma_{j}\right)$, for any $j$. Also, no component of $S_{j}$ is a compression disc for $R_{ \pm}\left(\gamma_{j}\right)$, since $\left(M_{j}, \gamma_{j}\right)$ is taut. In Part (a), $\left(M_{L}(r), \gamma\right)$ is not taut, and so Theorem 3.6 of [9] implies that $\left(M_{i}^{\prime}, \gamma_{i}\right)$ is not taut.

If $\left(M_{i}^{\prime}, \gamma_{i}\right)$ is taut, then by Theorem 3.6 of [9] and Corollary 3.3 of [9], each decomposing surface $S_{j}$ is taut in $M_{j}^{\prime}$, for $1 \leq j \leq i-1$. As noted in the argument of Theorem A.6, this implies that the map $i_{*}: \pi_{1}\left(V_{L}(r)\right) \rightarrow \pi_{1}\left(M_{L}(r)\right)$ induced by inclusion is an injection. Hence, in Part (b), if $\left(M_{i}^{\prime}, \gamma_{i}\right)$ is taut, then $\left[L^{\prime}\right]$ has finite order in $\pi_{1}\left(V_{L}(r)\right)$.

In Part (c), assume for the moment that $i \geq 2$. Now, $S_{1}$ is taut, by Corollary 3.3 of [9]. By Lemma A.7, $S_{1}$ is a norm minimising representative of $z_{\mu}$, and hence $z$. Also, since the Thurston norm of $z_{r}$ is less than that of $z_{\mu}$ or $z$, then $S_{1}$ is not taut in $\left(M_{1}^{\prime}, \gamma_{1}\right)$. Corollary 3.3 of [9] then implies that $\left(M_{2}^{\prime}, \gamma_{2}\right)$ is not taut. Theorem 3.6 of [9] implies that $\left(M_{i}^{\prime}, \gamma_{i}\right)$ is not taut. This proves the claim.

Case 1. $H_{2}\left(V_{L}, \partial V\right) \neq 0$ and $V \notin \mathcal{S}$, and at least one of the following holds:

(i) Part (a) or Part (b) applies, or

(ii) $i \neq 1$.

Let $F$ be $S_{i} \cap V$. It is argued in Lemma 2.4 that no component of $S_{i}$ is a sphere or disc disjoint from $\gamma_{i}$. Apply Theorem A.10 (a) (with $M$ and $\gamma$ of Theorem A.10 being $V$ and $\gamma_{i} \cap V$ ) to deduce there is an ambient isotopy of $L$ in $V$ such that after the isotopy, we have for each component $S_{i}^{j}$ of $F$

$$
\left|L \cap S_{i}^{j}\right| \leq \frac{-2 \chi\left(S_{i}^{j}\right)+\left|S_{i}^{j} \cap \gamma_{i}\right|}{2(\Delta(r, \mu)-1)} .
$$

The right-hand side is less than one, since we are assuming that $\Delta(r, \mu)>I(\mathcal{H})$. This proves the inductive step in this case.

Case 2. $H_{2}\left(V_{L}, \partial V\right) \neq 0$ and $V \notin \mathcal{S}$ and Part (c) applies and $i=1$.

Then $V=M$. Let $S$ be some taut conditioned surface properly embedded in $M_{L}$, which is disjoint from $\partial \mathcal{N}(L)$, which has no closed separating component, and such that $[S, \partial S]=z \in H_{2}\left(M_{L}, \partial M\right)$. Now, the Thurston norm of $z_{r}$ is less than that of $z$ or $z_{\mu}$, and so must be less than that of $z$. Hence, by Lemma A.7, $S$ is not taut in $M_{L}(r)$. Now apply Theorem A.10 (b) (with $F=S_{1}$ ). The inductive step follows as in Case 1. 
Case 3. $H_{2}\left(V_{L}, \partial V\right) \neq 0$ and $V \in \mathcal{S}$.

Then $L$ is homotopically trivial in $V$ and hence in $M$. In Parts (a) and (b), this contradicts Theorem A.9 (with $(N, \gamma)=\left(M_{L}, \gamma\right)$ and $P=\partial \mathcal{N}(L)$ ). In Part (c), it contradicts Corollary A.8.

Case 4. $H_{2}\left(V_{L}, \partial V\right)=0$.

Then $V_{L}$ is a J-cobordism on $\partial \mathcal{N}(L)$, and so is a rational homology cobordism by Lemma A.4 (d). So, $V_{L}$ is homeomorphic to $\partial \mathcal{N}(L) \times I$. Hence, $V \in \mathcal{S}$, and $L$ is a core of $V$. Now, $\partial V$ contains some sutures, since $\left(M_{i}, \gamma_{i}\right)$ is taut. Let $w^{\prime}$ be the winding number of a single suture in $V$. Then $w^{\prime} \leq w(\mathcal{H})$, where $w(\mathcal{H})$ is defined in 2.3. Now, $\left[L^{\prime}\right]$ has infinite order in $\pi_{1}\left(V_{L}(r)\right)$ and $i \geq 2$. Hence, by the claim, $\left(V_{L}(r), \gamma_{i} \cap \partial V\right)$ is not taut. This implies that $\Delta(r, \mu)=w^{\prime}$. Hence, $\Delta(r, \mu)=w^{\prime} \leq w(\mathcal{H}) \leq I(\mathcal{H})$. This is a contradiction, since we are assuming that $\Delta(r, \mu)>I(\mathcal{H})$.

Thus, the induction is complete. This proves the theorem.

The following result is the next step in proving Theorem 2.9. Note that the hypothesis in Theorem 2.5 about tori in $M_{L}$ has been replaced by an assumption about tori in $M$. Significant use is made of the results in the appendix.

Theorem 2.7. Let $(M, \gamma)$ be a connected taut sutured manifold. Let $L$ be a knot in $M$ which does not lie in a 3-ball. Suppose that $H_{2}\left(M_{L}, \partial M\right)$ is non-trivial. Let $\mu$ be the meridional slope on $\partial \mathcal{N}(L)$ and let $r$ be another slope. Suppose that every incompressible torus in $M$ which bounds a rational homology solid torus is parallel to a component of $\partial M$.

(a) If $\left(M_{L}(r), \gamma\right)$ is not taut, then $\Delta(r, \mu) \leq I(M, \gamma)$. In particular, if $M_{L}(r)$ is reducible, then $\Delta(r, \mu) \leq I(M)$.

(b) If the core of the surgery solid torus in $M_{L}(r)$ has finite order in $\pi_{1}\left(M_{L}(r)\right)$, then $\Delta(r, \mu) \leq I(M)$.

(c) Suppose that $\partial M$ is a (possibly empty) collection of tori and that $\gamma=\emptyset$. Let $z$ be a non-trivial element of $H_{2}\left(M_{L}, \partial M\right)$. Let $z_{\mu}$ and $z_{r}$ be the elements of $H_{2}(M, \partial M)$ and $H_{2}\left(M_{L}(r), \partial M_{L}(r)\right)$, respectively, to which $z$ is sent by the maps induced by inclusion. If the Thurston norm of $z_{r}$ is less than that of $z$ or $z_{\mu}$, then $\Delta(r, \mu) \leq I\left(M, \gamma, z_{\mu}\right)$

Proof. Let $H$ be a rational homology cobordism in $M_{L}$ between $\partial \mathcal{N}(L)$ and a properly embedded torus $T$. By Lemma A.15 (with $N=M_{L}$ and $P=\partial \mathcal{N}(L)$ and $F=\partial M), T$ is incompressible in $M_{L}$. By Haken's finiteness theorem [3, Lemma 13.2], we may assume that the only tori in $X=M_{L}-\operatorname{int}(H)$ which are cobordant to $T$ by a rational homology cobordism are parallel to $T$.

Note that $H(\mu)$ is a rational homology solid torus in $M$. T cannot be parallel to a component of $\partial M$, since then $H_{2}\left(M_{L}, \partial M\right) \cong H_{2}(H, T)$, which is trivial. Thus, by assumption, $T$ must be compressible in $M$. So, there is a ball $D^{2} \times I$ embedded in $M$ such that $\left(D^{2} \times I\right) \cap T=\partial D^{2} \times I$ and $\partial D^{2} \times\{0\}$ is essential in $T$. Then $T \cup\left(D^{2} \times \partial I\right)-\left(\partial D^{2} \times \operatorname{int}(I)\right)$ is a sphere which bounds a ball $B$ in $M$, since $M$ is irreducible. Since $T$ is incompressible in $M_{L}, D^{2} \times I$ lies in $H(\mu)$. If $B$ contains $D^{2} \times I$, then $X$ is homeomorphic to the exterior of a knot in $S^{3}$. This implies that $H_{2}(X, \partial M)$ is trivial. But, by Lemma A.14 (with $N=M_{L}$ and $P=\partial \mathcal{N}(L)$ and $F=\partial M)$, the map $i_{*}: H_{2}(X, \partial M) \rightarrow H_{2}\left(M_{L}, \partial M\right)$ induced by inclusion is an isomorphism. However, $H_{2}\left(M_{L}, \partial M\right)$ is, by assumption, non-trivial. This is a contradiction. Thus $B$ does not contain $D^{2} \times I$ and so $H(\mu)$ is a solid torus in $M$. 
Claim. $H(r)$ is either a solid torus or reducible.

This follows from Lemma A.12 (with $Q=M_{L}(r)$ and $Y=H(r)$ ) when $M_{L}(r)$ is reducible. If $R_{ \pm}\left(M_{L}(r)\right.$ ) is not taut in $M_{L}(r)$, Lemma A.18 (with $N=M_{L}$ and $\alpha=r$ and $\left.S=R_{ \pm}(M)\right)$ implies that $T$ compresses in $H(r)$, which proves the claim for Part (a).

In Part (b), the claim follows from Lemma A.19 (with $N=M_{L}$ and $P=\partial \mathcal{N}(L)$ and $\alpha=r)$.

In Part (c), we let $S$ be a taut representative of $z$ in $H_{2}\left(M_{L}, \partial M\right)$. By Lemma A.17 (with $N=M_{L}$ and $P=\partial \mathcal{N}(L)$ ), we may assume that $S$ is disjoint from $H$. (Thus, if $z^{\prime}$ is the inverse image of $z$ under the isomorphism from $H_{2}(X, \partial M)$ to $H_{2}\left(M_{L}, \partial M\right)$, then Lemma A.7 implies that the Thurston norm of $z^{\prime}$ is equal to that of $z$.) Assume further that $S$ has no closed separating components. Since the Thurston norm of $z_{r}$ is less than that of $z$ or $z_{\mu}$, it must be less than that of $z$. Lemma A.7 then implies $S$ is not taut in $M_{L}(r)$. Then Lemma A.18 (with $N=M_{L}$ and $\alpha=r$ ) implies that $H(r)$ is either a solid torus or reducible. This proves the claim.

Case 1. $H(r)$ is a solid torus.

Then the operation of removing $H(\mu)$ and inserting $H(r)$ is Dehn surgery. Thus, we may instead consider this surgery instead of surgery along $L$. The only tori in $M-\operatorname{int}(H(\mu))$ which are cobordant to $T$ by a rational homology cobordism are parallel to $T$. Let $\mu_{1}$ and $r_{1}$ be the slopes on $T$ which are trivial in $H_{1}(H(\mu) ; \mathbb{Q})$ and $H_{1}(H(r) ; \mathbb{Q})$, respectively. In Part (a), Theorem 2.5 (a) states that $\Delta\left(r_{1}, \mu_{1}\right) \leq$ $I(M, \gamma)$. In Part (b), Lemma A.19 (with $N=M_{L}$ and $P=\partial \mathcal{N}(L)$ and $\alpha=r$ ) implies that the core of $H(r)$ has finite order in $\pi_{1}\left(M_{L}(r)\right)$. Theorem 2.5 (b) then implies that $\Delta\left(r_{1}, \mu_{1}\right) \leq I(M)$. For Part (c), note that the Thurston norms of $z^{\prime}$ and $z$ are equal. Hence, the Thurston norm of $z_{r}$ is less than that of $z^{\prime}$ or $z_{\mu}$. Theorem 2.5 (c) then states that $\Delta\left(r_{1}, \mu_{1}\right) \leq I\left(M, \gamma, z_{\mu}\right)$. But Lemma A.20 (with $V=H(\mu))$ states that $\Delta(r, \mu) \leq \Delta\left(r_{1}, \mu_{1}\right)$, which proves the theorem in this case.

Case 2. $H(r)$ is reducible.

Then Corollary 4.4 of [10] implies that $\Delta(r, \mu)=1$. This proves the theorem.

We now consider the case where there may be some essential tori in $M$ which bound rational homology solid tori. The following result is presumably well known.

Theorem 2.8. Let $M$ be a compact, orientable, irreducible 3-manifold, possibly with boundary. Then there is a finite number of incompressible tori properly embedded in $M$, where we identify two tori if there is a homeomorphism of $M$ to itself which takes one to the other.

Proof. We may assume that $M$ is connected. By boundary-reducing $M$ if necessary, we may assume that $M$ is boundary-irreducible. If $M$ contains any incompressible tori, it is Haken. Thus, using the terminology of [4], there exists a characteristic Seifert pair $(\Sigma, \Phi) \subset(M, \partial M)$ such that each component $X$ of $\Sigma$ is Seifert fibred or an $I$-bundle over a compact surface. If $T$ is any incompressible torus in the interior of $M$, then there exists an isotopy of $M$ which takes $T$ into the interior of some component $X$ of $\Sigma$. Thus, it suffices to consider the set of incompressible tori in the interior of $X$, where we identify two tori if there is a homeomorphism of $X$ to itself which is isotopic to the identity on $\partial X$ and which sends one torus to the other. For, such a homeomorphism extends to a homeomorphism of $M$ to itself. 
Case 1. $X$ is an $I$-bundle over a compact surface $F$.

Now, the map $i_{*}: \pi_{1}(T) \rightarrow \pi_{1}(X)$ induced by inclusion is an injection. But $\pi_{1}(X) \cong \pi_{1}(F)$ and so $\pi_{1}(T)$ can be identified with a subgroup of $\pi_{1}(F)$. This corresponds to a covering of $F$. If this covering is infinite, then the group associated with it is the fundamental group of a non-compact surface, which is free. This is a contradiction, and so the covering is finite. Thus, by consideration of Euler characteristics, $F$ is a torus or Klein bottle. But, in these cases, $X$ is also Seifert fibred. Thus, we need only consider the following case.

Case 2. $X$ is Seifert fibred.

By Theorem VI.34 of [4], one of the following holds.

(i) $T$ is a fibre in a fibration of $X$ as a torus bundle over $S^{1}$, or

(ii) $X=X_{1} \cup X_{2}$, where $X_{1} \cap X_{2}=\partial X_{1}=\partial X_{2}=T$, and, for each $i, X_{i}$ is the orientable twisted $I$-bundle over a non-orientable surface $F_{i}$ (possibly with boundary), or

(iii) $T$ is a union of fibres in some Seifert fibration of $X$.

Note that in case (iii), $T$ must in fact be a union of regular fibres.

In case (i), the fibration determines a homeomorphism $X \cong S^{1} \times S^{1} \times I / \phi$, where $\phi: S^{1} \times S^{1} \times\{0\} \rightarrow S^{1} \times S^{1} \times\{1\}$ is some homeomorphism. Let $h \in \pi_{1}(X)$ be represented by a regular fibre of the Seifert fibration. It is argued in Theorem VI.34 of [4] that, if case (iii) does not hold, then $\langle h\rangle \nless \pi_{1}(T)$. By VI.26 of [4], we may take $\phi$ to be periodic. By the argument of Lemma 12.4 of [3] and Examples 12.3 of [3], such a $\phi$ is unique up to isotopy, for a given $X$. Thus, if two tori in $X$ satisfy (i) but not (iii), then there is a homeomorphism of $X$ which takes one to the other.

In case (ii), we must have $\chi\left(F_{i}\right)=0$. Since the orientable twisted $I$-bundle over the Möbius band is a solid torus, each $F_{i}$ is a Klein bottle. Suppose that $T_{1}$ is another incompressible torus in $X$. Ambient isotope $T_{1}$ in $X$ so as to minimise $T_{1} \cap T$. If $T_{1}$ lies in some $X_{i}$, then Theorem VI.34 of [4] implies that $T_{1}$ is a union of fibres in some Seifert fibration of $X_{i}$. There are two possible Seifert fibrations of $X_{i}$ up to homeomorphism not necessarily fixed on $\partial X_{i}$ (see VI.5 (d) of [4]). The first Seifert fibration has base manifold the disc and two exceptional fibres. $T_{1}$ is union of regular fibres and so is determined by a simple closed curve in the twice-punctured disc. Since $T_{1}$ is incompressible, $T_{1}$ is parallel in this case to $T$. The second Seifert fibration has base manifold the Möbius band and no exceptional fibres. There are, up to homeomorphism, two simple closed curves in the interior of the Möbius band. The inverse image of one of these is a Klein bottle. Hence, $T_{1}$ must be the inverse image of the other and so be parallel to $T$.

If $T_{1}$ does not lie in some $X_{i}$, then $T_{1} \cap X_{i}$ is a union of annuli, for each $i$, and each annulus cannot be isotoped (rel its boundary) into $T$. It is then straightforward to deduce, from Theorem VI.34 and Example VI.5 (d) of [4], that the annuli are a union of fibres in a Seifert fibration of $X_{i}$. Hence, $T_{1}$ is a union of fibres in a Seifert fibration of $X$.

Thus, we may assume that case (iii) holds. We must now consider some 'exceptional' cases for $X . X$ is not a solid torus, for then $T$ would be compressible. If $X$ is homeomorphic to the orientable $I$-bundle over the Klein bottle, then we have already shown, in case (ii), that there is a unique incompressible torus $T$ in $X$ up to ambient isotopy. If $X$ is homeomorphic to $S^{1} \times S^{1} \times I$, then, by Theorem VI.17 of [4], there is a fibre-preserving homeomorphism (not necessarily fixed on $\partial X$ ) from 
$X$ to the product $S^{1}$-bundle over $S^{1} \times I$. Since $T$ is a union of fibres, it must be parallel to each component of $\partial X$. Thus, in this case, $T$ is unique up to an isotopy of $X$.

We can assume that $X$ is not one of the exceptional cases considered above. When $\partial X \neq \emptyset$, Theorem VI.18 of [4] implies that there is a unique Seifert fibration of $X$ up to ambient isotopy. When $\partial X=\emptyset$, Theorem VI.17 and Examples VI.16 of [4] imply that $X$ has a finite number of Seifert fibrations, up to fibre-preserving homeomorphism. $T$ is a union of fibres in one such Seifert fibration.

Let $F$ be the base space of $X$. Let $N$ be a fibred regular neighbourhood of the exceptional fibres, or a fibred regular neighbourhood of a single fibre if there are no exceptional fibres and $F$ is closed. Thus, $X_{1}=X-\operatorname{int}(N)$ is the total space of an $S^{1}$-bundle $\eta_{1}$ over a surface $F_{1}$ which is a subset of $F$. Let $P$ be some component of $\partial N$. Let $\eta_{2}$ (respectively, $\eta_{3}$ ) be an $S^{1}$-bundle obtained from $\eta_{1}$ by attaching to each component of $\partial X_{1}$ (respectively, $\partial X_{1}-P$ ) a product $S^{1}$-bundle over a 2-disc, in such a way that fibres are identified with fibres. Let $p_{2}: X_{2} \rightarrow F_{2}$ be the projection map for $\eta_{2}$. Thus, $F_{2}$ is obtained from $F_{1}$ by attaching a number of 2-discs. Now, $T$ is a union of regular fibres in $X$, and thus we may assume that $T$ lies in $X_{1}$, and hence $X_{2} \cdot p_{2}(T)$ is a simple closed curve in $F_{2}$. Up to homeomorphism of $F_{2}$ to itself which is supported in $F_{1}$, there is only a finite number of such curves. Such a homeomorphism $h: F_{2} \rightarrow F_{2}$ induces the bundle $h ! \eta_{2}$, with total space $X_{3}$, say, and projection map $p_{3}: X_{3} \rightarrow F_{2}$ :

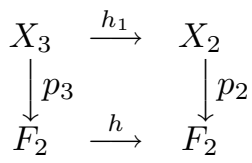

Now, $\eta_{2}$ is determined by two invariants [12]. The first is a homomorphism $\phi\left(\eta_{2}\right): \pi_{1}\left(F_{2}\right) \rightarrow \mathbb{Z}_{2}$. Since $X_{2}$ is orientable, this is the orientation homomorphism. The second is an element $b\left(\eta_{2}\right) \in H^{2}\left(F_{2} ; \mathbb{Z}\right)$, where the coefficient group $\mathbb{Z}$ is twisted by the map $\phi$. In our case, the resulting cohomology group is isomorphic to $\mathbb{Z}$. $b\left(\eta_{2}\right)$ can be interpreted as the intersection number in $P$ of the restriction of a section of $\eta_{3}$ and the boundary of a meridian disc. Thus, $b\left(h ! \eta_{2}\right)=b\left(\eta_{2}\right)$. Also, $\phi\left(h^{!} \eta_{2}\right)=\phi\left(\eta_{2}\right) \circ h_{*}=\phi\left(\eta_{2}\right)$. Hence, $h^{!} \eta_{2}$ is the same bundle as $\eta_{2}$. That is, there is an orientation-preserving bundle isomorphism:

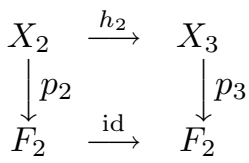

Now, $h_{1} \circ h_{2}$ restricts to a fibre-preserving homeomorphism of $X_{1}$ to itself, since $h$ was supported in $F_{1}$. It fixes the fibres and meridians of $\partial X_{1}$ up to isotopy, and hence is isotopic to the identity on $\partial X_{1}$. Hence, up to homeomorphism of $X$ to itself fixed on $\partial X$, there is a finite number of incompressible tori in the interior of $X$.

We are now in a position to prove one of the principal results of this paper.

Theorem 2.9. Let $M$ be a compact connected orientable irreducible 3-manifold, possibly with boundary. Let $L$ be a knot in $M$ which does not lie in a 3-ball. Suppose that $H_{2}\left(M_{L}, \partial M\right) \neq 0$. Let $\mu$ be the meridional slope on $\partial \mathcal{N}(L)$, and let $r$ be another slope. Suppose that at least one of the following holds. 
(a) There is a sphere in $M_{L}(r)$ which does not bound a rational homology 3-ball, or

(b) $M_{L}(r)$ is reducible, and every incompressible torus in $M$ which bounds a rational homology solid torus is parallel to a component of $\partial M$, or

(c) the core of the surgery solid torus in $M_{L}(r)$ has finite order in $\pi_{1}\left(M_{L}(r)\right)$.

Then there is an upper bound on $\Delta(r, \mu)$ which depends only on $M$.

Proof. Let $(M, \gamma)$ be a taut sutured manifold structure on $M$. This exists by Theorem 2.1. Part (b) immediately follows from Theorem 2.7 (a). We shall now prove Parts (a) and (c). Let $H$ be a rational homology cobordism in $M_{L}$ between $\partial \mathcal{N}(L)$ and a properly embedded torus $T$. Let $X=M_{L}-\operatorname{int}(H)$. We may assume that every torus in $X$ which is cobordant to $T$ by a rational homology cobordism is parallel to $T$. If $T$ is compressible in $M$, then the argument of Theorem 2.7 proves the theorem. Thus, we assume that $T$ is incompressible in $M$.

Let $\mu_{1}$ and $r_{1}$ be the slopes on $T$ which are trivial in $H_{1}(H(\mu) ; \mathbb{Q})$ and $H_{1}(H(r) ; \mathbb{Q})$ respectively. Note that $(X, \gamma)$ is a taut sutured manifold. Note also that $H_{2}(X, \partial M)$ is non-trivial by Lemma A.14 (with $N=M_{L}$ and $P=\partial \mathcal{N}(L)$ and $F=\partial M)$.

In Part (a), Lemma A.11 (with $Q=M_{L}(r)$ and $Y=H(r)$ and $\alpha_{1}=r_{1}$ ) states that $X\left(r_{1}\right)$ is reducible and that $T$ compresses in $H(r)$. In Part (c), Lemma A.19 (with $N=M_{L}$ and $P=\partial \mathcal{N}(L)$ and $\alpha=r$ ) implies that the core $L_{1}$ of the surgery solid torus in $X\left(r_{1}\right)$ has finite order in $\pi_{1}\left(X\left(r_{1}\right)\right)$, and that $T$ compresses in $H(r)$. So, in both Part (a) and Part (c), Theorem A.9 (with $N=X$ and $P=T$ ) implies that $\left(X\left(\mu_{1}\right), \gamma\right)$ is taut. Theorem 2.5 (a) or (b) (with $M$ and $L$ of Theorem 2.5 being $X\left(\mu_{1}\right)$ and the core of the surgery solid torus) states that there is an upper bound on $\Delta\left(\mu_{1}, r_{1}\right)$ which depends only on $X\left(\mu_{1}\right)$.

We wish to remove the dependence of the upper bound on $X\left(\mu_{1}\right)$. Theorem 2.8 states that there is a finite number of incompressible tori in the interior of $M$ up to homeomorphism of $M$. Hence, up to homeomorphism, there are only a finite number of possibilities for $X\left(\mu_{1}\right)$. Hence, we can find an upper bound on $\Delta\left(\mu_{1}, r_{1}\right)$ which depends only on $M$. Lemma A.20 (with $V=H(\mu)$ ) states that $K \Delta\left(r_{1}, \mu_{1}\right) \geq \Delta(r, \mu)$, for some constant $K$ which depends only on $H(\mu) \subset M$. Hence, we can find a constant $K$ which depends only on $M$. Thus, there is an upper bound on $\Delta(r, \mu)$ which depends only on $M$.

\section{AN EXAMPLE}

In this section, we consider the case where $M$ is $F \times S^{1}$, where $F$ is a compact connected orientable surface, possibly with boundary, which is neither a sphere nor a disc.

Lemma 3.1. There is no incompressible torus $T$ in $M$ which bounds a rational homology solid torus.

Proof. Now, $M$ is a compact orientable Seifert fibre space. The two-sided incompressible surfaces in such a manifold are classified in Theorem VI.34 of [4]. We deduce that such a torus $T$ is a union of fibres of some Seifert fibration of $M$. Theorem VI.17 of [4] implies that the pair $(M, T)$ is homeomorphic to $\left(F \times S^{1}, C \times S^{1}\right)$, where $C$ is an essential simple closed curve in $F$. For $T$ to be separating, $C$ must be separating. This implies that each component $X$ of $F-C$ has $\operatorname{rank}\left(H_{1}(X ; \mathbb{Q})\right) \geq 1$. Therefore, neither component of $M-T$ is a rational homology solid torus. 


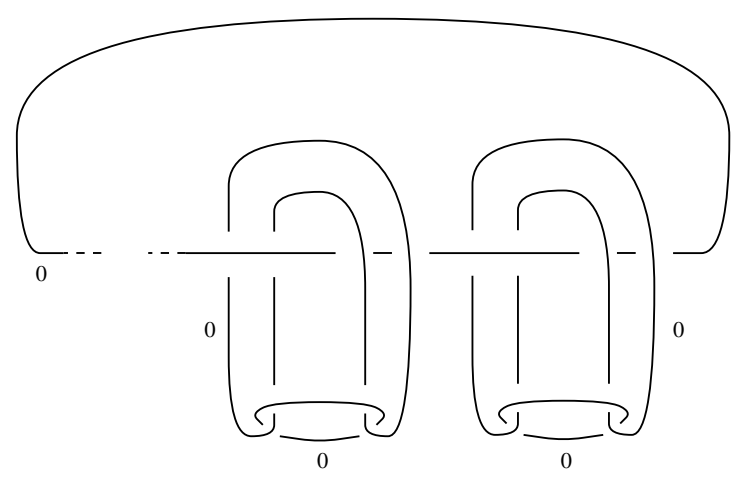

Figure 2

Note also that $\operatorname{rank}\left(H_{2}(M, \partial M ; \mathbb{Q})\right)=\operatorname{rank}\left(H_{1}(M ; \mathbb{Q})\right)=\operatorname{rank}\left(H_{1}(F ; \mathbb{Q})\right)+$ $\operatorname{rank}\left(H_{1}\left(S^{1} ; \mathbb{Q}\right)\right) \geq 2$. So, for any knot $L$ in $M, H_{2}\left(M_{L}, \partial M\right)$ is non-trivial.

We now construct a taut sutured manifold sequence for $M$. Let $\left(M_{1}, \gamma_{1}\right)=$ $(M, \emptyset)$. There is a (possibly empty) collection $\left\{C_{i}: i \in \mathcal{C}\right\}$ of disjoint simple closed curves in $F$ such that

(i) if $\chi(F)<0$, then $F_{2}=F-\operatorname{int}\left(\mathcal{N}\left(\bigcup_{i \in \mathcal{C}} C_{i}\right)\right)$ is a collection of 'pairs of pants', that is, planar surfaces each with three boundary components, and

(ii) if $\chi(F)=0$, then $F_{2}=F-\operatorname{int}\left(\mathcal{N}\left(\bigcup_{i \in \mathcal{C}} C_{i}\right)\right)$ is an annulus.

Let $S_{1}=\bigcup_{i \in \mathcal{C}} C_{i} \times S^{1}$. Then $-2 \chi\left(S_{1}^{j}\right)+\left|S_{1}^{j} \cap \gamma_{1}\right|=0$, for each component $S_{1}^{j}$ of $S_{1}$. Let $\left(M_{2}, \gamma_{2}\right)$ be the sutured manifold obtained by decomposing $\left(M_{1}, \gamma_{1}\right)$ along $S_{1}$. There is a collection $\left\{\alpha_{i}: i \in \mathcal{C}^{\prime}\right\}$ of disjoint properly embedded arcs in $F_{2}$ such that $F_{3}=F_{2}-\operatorname{int}\left(\mathcal{N}\left(\bigcup_{i \in \mathcal{C}^{\prime}} \alpha_{i}\right)\right)$ is a collection of discs. Each pair of pants contains two such $\alpha_{i}$, and an annulus contains one such $\alpha_{i}$. Let $S_{2}=\bigcup_{i \in \mathcal{C}^{\prime}} \alpha_{i} \times S^{1}$. Then $-2 \chi\left(S_{2}^{j}\right)+\left|S_{2}^{j} \cap \gamma_{2}\right|=0$, for each component $S_{2}^{j}$ of $S_{2}$. Let $\left(M_{3}, \gamma_{3}\right)$ be the sutured manifold obtained by decomposing $\left(M_{2}, \gamma_{2}\right)$ along $S_{2}$. Now, $M_{3}$ is a collection of solid tori. The winding number of each suture in each solid torus is 1 . Finally, let $S_{3}$ be the collection of discs $F_{3} \times\{*\}$, where $\{*\}$ is a point in $S^{1}$. Let $\left(M_{4}, \gamma_{4}\right)$ be the sutured manifold obtained by decomposing $\left(M_{3}, \gamma_{3}\right)$ along $S_{3}$. Then $\left(M_{4}, \gamma_{4}\right)$ is a collection of balls each with one suture in its boundary. This sutured manifold is taut. Therefore, by Theorem 3.6 of [9], this sutured manifold sequence $\mathcal{H}$ is taut. In particular $\left(M_{1}, \gamma_{1}\right)$ is taut. Now, the set $\mathcal{S}$ of Definition 2.3 is the union of the components of $M_{3}$. Hence, $w(\mathcal{H})=1$. Therefore, $I(\mathcal{H})=1$ and so $I(M) \leq 1$. In fact, by Lemma $2.4, I(M)=1$. Thus, Theorem 2.7 implies the following result.

Theorem 3.2. Let $M$ be $F \times S^{1}$, where $F$ is a compact connected orientable surface, possibly with boundary, which is neither a sphere nor a disc. Let $L$ be a knot in $M$ which does not lie in a 3-ball. Let $\mu$ be the meridional slope on $\partial \mathcal{N}(L)$, and let $r$ be another slope. If $M_{L}(r)$ is reducible or the core of the surgery solid torus has finite order in $\pi_{1}\left(M_{L}(r)\right)$, then $\Delta(r, \mu)=1$.

Examples of such $L$ exist. If $L$ is cabled and $r$ is the slope of the boundary of the cabling annulus, then $M_{L}(r)$ is reducible. Another set of examples is the following. Figure 2 is a surgery diagram for $F \times S^{1}$, where $F$ is a closed connected orientable surface which is not a sphere. Let $L$ be the core of the surgery solid torus corresponding to any of the curves in Figure 2. Then, re-inserting this solid 
torus via the slope which was meridional in $S^{3}$ yields a manifold with an $S^{2} \times S^{1}$ summand.

\section{Distinguishing 3-MANifolds obtained By Dehn SURgery}

The main goal of this section will be to prove the following two theorems.

Theorem 4.1. For $i=1$ and 2 , let $V^{i}$ be a compact connected oriented 3-manifold, possibly with boundary, such that $H_{1}\left(V^{i} ; \mathbb{Q}\right) \neq 0$. Let $K_{i}$ be a homotopically trivial knot in $V^{i}$, such that $V^{i}-K_{i}$ is irreducible and atoroidal. Let $V_{K_{i}}^{i}\left(p_{i} / q_{i}\right)$ be the manifold obtained from $V^{i}$ by $p_{i} / q_{i}$ Dehn surgery along $K_{i}$, where $p_{i}$ and $q_{i}$ are coprime integers. Then, there is a natural number $C\left(V^{1}, K_{1}\right)$, which depends only on $V^{1}$ and $K_{1}$, with the following property. If $\left|q_{2}\right|>C\left(V^{1}, K_{1}\right)$, then

$$
V_{K_{1}}^{1}\left(p_{1} / q_{1}\right) \cong V_{K_{2}}^{2}\left(p_{2} / q_{2}\right) \Longleftrightarrow\left\{\begin{array}{c}
\left(V^{1}, K_{1}\right) \cong\left(V^{2}, K_{2}\right) \\
\text { and } \\
p_{1} / q_{1}=p_{2} / q_{2}
\end{array}\right\},
$$

where $\cong$ denotes an orientation-preserving homeomorphism.

Theorem 4.2. Let $V^{1}$ and $K_{1}$ be as in Theorem 4.1. If $\left|q_{1}\right| \geq C\left(V^{1}, K_{1}\right)$, then $V_{K_{1}}^{1}\left(p_{1} / q_{1}\right)$ is irreducible, and $I\left(V_{K_{1}}^{1}\left(p_{1} / q_{1}\right)\right)=\left|q_{1}\right|$.

We must introduce some new invariants which are closely related to $I(M)$.

Definition 4.3. Let $(N, \gamma)$ be a connected taut sutured manifold. Let $P$ be a toral component of $\partial N$. Suppose that $H_{2}(N, \partial N-P)$ is non-trivial and that $z$ is an element of $H_{2}(N, \partial N-P)$. Suppose that the only tori in $N$ which are cobordant to $P$ by a rational homology cobordism are parallel to $P$. Let $\mathcal{H}$ be a taut sutured manifold sequence

$$
\left(N_{1}, \gamma_{1}\right) \stackrel{S_{1}}{\longrightarrow}\left(N_{2}, \gamma_{2}\right) \stackrel{S_{2}}{\longrightarrow} \ldots \stackrel{S_{n-1}}{\longrightarrow}\left(N_{n}, \gamma_{n}\right),
$$

such that

(i) $\left(N_{1}, \gamma_{1}\right)=(N, \gamma)$, and

(ii) $\left[S_{1}, \partial S_{1}\right]=z \in H_{2}(N, \partial N-P)$, and

(iii) no component of $\partial S_{i}$ bounds a disc in $R_{ \pm}\left(\gamma_{i}\right)$, for any $i$, and

(iv) $S_{i} \cap P=\emptyset$, for each $i$, and

(v) $N_{n}$ is a collar $H$ on $P$, together with a collection of balls, and

(vi) $\gamma_{n} \cap(\partial H-P) \neq \emptyset$.

If $z \neq 0$, then such a sequence always exists by the arguments of Theorem 5.1 of [9] together with Theorem A.6 of this paper. Let $\mathcal{S}$ be the set of components of $\bigcup_{i=1}^{n} N_{i}$ which are solid tori. Let $w(\mathcal{H})$ be the maximum winding number of any single suture in an element of $\mathcal{S}$. Let $w(\mathcal{H})=0$ if $\mathcal{S}=\emptyset$. Let $\left\{S_{i}^{j}: j \in \mathcal{C}_{i}\right\}$ be the set of components of $S_{i}$ which are not in an element of $\mathcal{S}$. Let

$$
\tilde{I}(\mathcal{H})=\max \left\{\max _{1 \leq i \leq n-1} \max _{j \in \mathcal{C}_{i}}\left\{-\chi\left(S_{i}^{j}\right)+\frac{\left|S_{i}^{j} \cap \gamma_{i}\right|}{2}+1\right\}, w(\mathcal{H})\right\} .
$$

In the above, we follow the convention that the maximum value of an empty set of integers is zero. Define $\tilde{I}(N, \gamma, P, z)$ to be the minimum of $\tilde{I}(\mathcal{H})$ over all such sequences $\mathcal{H}$. If $z=0$ and there is no such sequence, we define $\tilde{I}(N, \gamma, P, z)$ to be infinite. Define $\tilde{I}(N, \gamma, P)$ to be the minimum of $\tilde{I}(N, \gamma, P, z)$ over all $z$. Define 
$\tilde{I}(N, P)$ to be the minimum of $\tilde{I}(N, \gamma, P)$ over all possible taut sutured manifold structures on $N$.

A sample calculation of $\tilde{I}(\mathcal{H})$ for a suitable sequence $\mathcal{H}$ is given in Example 4.9.

Theorem 4.4. Let $N$ be a compact connected orientable irreducible 3-manifold. Let $\alpha$ and $r$ be distinct slopes on a toral component $P$ of $\partial N$. Suppose that $H_{2}(N, \partial N-P) \neq 0$, and that the only tori in $N$ which are cobordant to $P$ by a rational homology cobordism are parallel to $P$. Suppose also that $N(r)$ is reducible or that the core of the surgery solid torus has finite order in $\pi_{1}(N(r))$. Then, $N(\alpha)$ is irreducible and one of the following inequalities holds:

$$
\begin{gathered}
\tilde{I}(N, P)<I(N(\alpha))=\Delta(r, \alpha), \\
\Delta(r, \alpha) \leq I(N(\alpha)) \leq \tilde{I}(N, P) .
\end{gathered}
$$

Hence, if $\Delta(r, \alpha) \geq \tilde{I}(N, P)$, then $I(N(\alpha))=\Delta(r, \alpha)$.

Proof. Let $(N, \gamma)$ be a taut sutured manifold structure, which exists by Theorem 2.1. Let $\mathcal{H}$ be a sutured manifold sequence as in Definition 4.3. We may assume that $\tilde{I}(\mathcal{H})=\tilde{I}(N, \gamma, P)=\tilde{I}(N, P)$. Note that no component of $\partial S_{i}$ bounds a disc in $R_{ \pm}\left(\gamma_{i}\right)$, for any $i$, and that no component of $S_{i}$ is a compression disc for $R_{ \pm}\left(\gamma_{i}\right)$. Hence, Theorem 3.6 of [9] and the argument of Theorem A.6 imply that if $\left(N_{n}\left(r^{\prime}\right), \gamma_{n}-P\right)$ is taut for some slope $r^{\prime}$ on $P$, then $\left(N\left(r^{\prime}\right), \gamma-P\right)$ is taut and the core of the surgery solid torus in $N\left(r^{\prime}\right)$ has infinite order in $\pi_{1}\left(N\left(r^{\prime}\right)\right)$. Therefore, $\left(N_{n}(r), \gamma_{n}-P\right)$ is not taut, and so the sutures in $\gamma_{n} \cap(\partial H-P)$ are parallel to $r$. By Dehn filling each manifold of the sequence along the slope $\alpha$, we obtain a taut sutured manifold sequence $\mathcal{H}_{1}$ for $(N(\alpha), \gamma-P)$. The final term of this sequence is the solid torus $H(\alpha)$, together with a collection of balls. Let $\mathcal{H}_{1}^{+}$be the sequence $\mathcal{H}_{1}$ with one further taut decomposition of the solid torus along a meridian disc. Then, $\mathcal{H}_{1}^{+}$satisfies all the requirements of Definition 2.3 (with $\left(M_{1}, \gamma_{1}\right)=(N(\alpha), \gamma-P)$ and $\left.z_{\mu}=\left[S_{1}, \partial S_{1}\right]\right)$.

Claim. $I(N(\alpha)) \leq I\left(\mathcal{H}_{1}^{+}\right) \leq \max \{\tilde{I}(N, P), \Delta(r, \alpha)\}$.

The first inequality is clear. We shall therefore concentrate on the second. Let $\mathcal{S}\left(\mathcal{H}_{1}^{+}\right)$and $\mathcal{S}(\mathcal{H})$ be the union of the solid toral components of the sutured manifolds of $\mathcal{H}_{1}^{+}$and $\mathcal{H}$, respectively. Let $\mathcal{C}_{i}\left(\mathcal{H}_{1}^{+}\right)$(respectively, $\mathcal{C}_{i}(\mathcal{H})$ ) be the sets $\mathcal{C}_{i}$ defined in 4.3 (respectively, 2.3) for the sequence $\mathcal{H}_{1}^{+}$(respectively, $\mathcal{H}$ ). Then $\mathcal{S}(\mathcal{H}) \subset \mathcal{S}\left(\mathcal{H}_{1}^{+}\right)$, and so there is a natural inclusion of $\bigcup_{i=1}^{n} \mathcal{C}_{i}\left(\mathcal{H}_{1}^{+}\right)$into $\bigcup_{i=1}^{n-1} \mathcal{C}_{i}(\mathcal{H})$. It therefore suffices to show that

$$
w\left(\mathcal{H}_{1}^{+}\right) \leq \max \{w(\mathcal{H}), \Delta(r, \alpha)\} .
$$

To do this, we must consider a solid torus $V$ in $\mathcal{S}\left(\mathcal{H}_{1}^{+}\right)-\mathcal{S}(\mathcal{H})$. We shall show that the winding number of a suture in $V$ is $\Delta(r, \alpha)$. The surgery solid torus must lie in $V$. Let $L$ be the core of this surgery solid torus. If $H_{2}\left(V_{L}, \partial V\right) \neq 0$, then $L$ is homotopically trivial in $V$ and hence $N(\alpha)$. This contradicts Theorem A.9. Thus, $H_{2}\left(V_{L}, \partial V\right)=0$. Therefore, $V_{L}$ is a J-cobordism on $\partial \mathcal{N}(L)$ and so a rational homology cobordism by Lemma A.4 (d). By assumption, it is a collar on $\partial \mathcal{N}(L)$. We deduce that the winding number of a suture in $V$ is $\Delta(r, \alpha)$. This proves the claim. 
Now, $N(r)$ is reducible or the core of the surgery solid torus has finite order in $\pi_{1}(N(r))$. Hence, by Theorem 2.5 (a) or (b) (with $(M, \gamma)$ and $L$ of 2.5 being $(N(\alpha), \gamma-P)$ and the core of the surgery solid torus)

$$
\Delta(r, \alpha) \leq I(N(\alpha)) .
$$

The above inequality and the claim prove the theorem.

Thus, if $\Delta(r, \alpha)$ is sufficiently large, then the invariant $I(N(\alpha))$ encodes $\Delta(r, \alpha)$. Theorem 4.4 also demonstrates that the upper bounds in Theorem 2.5 (a) and (b) are often achieved.

Proof of Theorem 4.2. In this proof, we may take $C\left(V^{1}, K_{1}\right)$ to be any integer greater than or equal to $\tilde{I}\left(V_{K_{1}}^{1}, \partial \mathcal{N}\left(K_{1}\right)\right)$. (See the proof of Theorem 4.1 for an actual definition of $C\left(V^{1}, K_{1}\right)$.) Note that, since $K_{1}$ is homotopically trivial in $V^{1}$, $\operatorname{rank}\left(H_{2}\left(V_{K_{1}}^{1}, \partial V^{1} ; \mathbb{Q}\right)\right)=\operatorname{rank}\left(H_{2}\left(V^{1}, \partial V^{1} ; \mathbb{Q}\right)\right)=\operatorname{rank}\left(H_{1}\left(V^{1} ; \mathbb{Q}\right)\right)>0$. Thus, $H_{2}\left(V_{K_{1}}^{1}, \partial V^{1}\right)$ is non-trivial. Hence, by Lemma A.15 (with $N=V_{K_{1}}^{1}$ and $P=$ $\partial \mathcal{N}\left(K_{1}\right)$ and $\left.F=\partial V^{1}\right)$ and the assumption that $V^{1}-K_{1}$ is atoroidal, the only tori in $V_{K_{1}}^{1}$ which are cobordant to $\partial \mathcal{N}\left(K_{1}\right)$ by a rational homology cobordism are parallel to $\partial \mathcal{N}\left(K_{1}\right)$. Theorem 4.4 (with $N=V_{K_{1}}^{1}$ and $P=\partial \mathcal{N}\left(K_{1}\right)$ and $r=\infty$ and $\left.\alpha=p_{1} / q_{1}\right)$ gives the result.

We can use Theorem 4.4 to prove that $V_{K_{1}}^{1}\left(p_{1} / q_{1}\right)$ and $V_{K_{2}}^{2}\left(p_{2} / q_{2}\right)$ are distinct if $\left|q_{1}\right| \neq\left|q_{2}\right|$ and $\left|q_{2}\right|$ is sufficiently large. However, Theorem 4.1 is a good deal stronger than this.

There are other versions of Theorem 4.4. For example, the following theorem has an entirely analogous proof.

Theorem 4.5. Let $N$ be a compact connected orientable irreducible 3-manifold with boundary a non-empty collection of tori. Let $\alpha$ and $r$ be distinct slopes on a component $P$ of $\partial N$. Suppose that the only tori in $N$ which are cobordant to $P$ by a rational homology cobordism are parallel to $P$. Let $z$ be a non-trivial element of $H_{2}(N, \partial N-P)$. Let $z_{\alpha}$ (respectively, $z_{r}$ ) be the element in $H_{2}(N(\alpha), \partial N(\alpha))$ (respectively, $H_{2}(N(r), \partial N(r))$ ) to which $z$ is sent by the map induced by inclusion. Suppose the Thurston norm of $z_{r}$ is less than that of $z$ or $z_{\alpha}$. Then, $(N(\alpha), \emptyset)$ is taut and one of the following inequalities holds:

$$
\begin{gathered}
\tilde{I}(N, \emptyset, P, z)<I\left(N(\alpha), \emptyset, z_{\alpha}\right)=\Delta(r, \alpha), \\
\Delta(r, \alpha) \leq I\left(N(\alpha), \emptyset, z_{\alpha}\right) \leq \tilde{I}(N, \emptyset, P, z) .
\end{gathered}
$$

Hence, if $\Delta(r, \alpha) \geq \tilde{I}(N, \emptyset, P, z)$, then $I\left(N(\alpha), \emptyset, z_{\alpha}\right)=\Delta(r, \alpha)$.

The following result is the key step in the proof of Theorem 4.1.

Theorem 4.6. Let $M$ be a compact connected orientable irreducible 3-manifold, possibly with boundary. For $i=1$ and 2 , suppose the following is true. Let $L_{i}$ be a knot in $M$ which does not lie in a 3-ball. Suppose that $H_{2}\left(M_{L_{i}}, \partial M\right) \neq$ 0 , and that the only tori in $M_{L_{i}}$ which are cobordant to $\partial \mathcal{N}\left(L_{i}\right)$ by a rational homology cobordism are parallel to $\partial \mathcal{N}\left(L_{i}\right)$. Let $\mu_{i}$ be the meridional slope on $\partial \mathcal{N}\left(L_{i}\right)$. Suppose that, for some other slope $r_{i}$ on $\partial \mathcal{N}\left(L_{i}\right), M_{L_{i}}\left(r_{i}\right)$ is reducible or the core of the surgery solid torus has finite order in $\pi_{1}\left(M_{L_{i}}\left(r_{i}\right)\right)$. Suppose also that $\Delta\left(r_{2}, \mu_{2}\right)>\tilde{I}\left(M_{L_{1}}, \partial \mathcal{N}\left(L_{1}\right)\right)$. Then, there is an isotopy of $M$ which takes $L_{2}$ to $L_{1}$, and $r_{2}$ to $r_{1}$. 
Proof. Let $\left(M_{L_{1}}, \gamma\right)$ be a taut sutured manifold structure, which exists by Theorem 2.1. Let $\mathcal{H}$ be a sutured manifold sequence for $\left(M_{L_{1}}, \gamma\right)$, as in Definition 4.3 (with $(N, \gamma)=\left(M_{L_{1}}, \gamma\right)$ and $\left.P=\partial \mathcal{N}\left(L_{1}\right)\right)$. We may assume that $\tilde{I}(\mathcal{H})=$ $\tilde{I}\left(M_{L_{1}}, \gamma, \partial \mathcal{N}\left(L_{1}\right)\right)=\tilde{I}\left(M_{L_{1}}, \partial \mathcal{N}\left(L_{1}\right)\right)$. Let $\mathcal{H}_{1}^{+}$be the sequence obtained from $\mathcal{H}$ as in Theorem 4.4. That is, we Dehn fill each manifold of the sequence via the slope $\mu_{1}$ on $\partial \mathcal{N}\left(L_{1}\right)$, and then extend the sequence by one further decomposition. Let $\mathcal{H}_{1}^{+}$be

$$
\left(M_{1}, \gamma_{1}\right) \stackrel{S_{1}}{\longrightarrow}\left(M_{2}, \gamma_{2}\right) \stackrel{S_{2}}{\longrightarrow} \ldots \stackrel{S_{n}}{\longrightarrow}\left(M_{n+1}, \gamma_{n+1}\right),
$$

where $\left(M_{1}, \gamma_{1}\right)=(M, \gamma)$ and $M_{n+1}$ is a collection of balls. It is argued in Theorem 4.4 that $\mathcal{H}_{1}^{+}$is a taut sutured manifold sequence.

Claim. There is an ambient isotopy of $L_{2}$ in $M$ such that, after the isotopy:

(a) $L_{2}$ lies in the same component of $M_{j}$ as $L_{1}$, for some $j$, and

(b) this component is a solid torus, of which $L_{2}$ is a core.

The proof of this is similar to that of Theorem 2.5. Suppose that $L_{2}$ lies in $M_{i}$, but that the component $V$ of $M_{i}$ containing $L_{2}$ is not of the required form. We shall prove that we can ambient isotope $L_{2}$ in $V$, so that after the isotopy $L_{2}$ lies in $M_{i+1}$. This will prove the claim, for $L_{2}$ cannot lie in $M_{n+1}$.

Note that, by the argument of the claim in the proof of Theorem 2.5, $\left(V_{L_{2}}\left(r_{2}\right)\right.$, $\left.\gamma_{i} \cap \partial V\right)$ is not taut or the core of the surgery solid torus in $V_{L_{2}}\left(r_{2}\right)$ has finite order in $\pi_{1}\left(V_{L_{2}}\left(r_{2}\right)\right)$.

Case 1. $H_{2}\left(V_{L_{2}}, \partial V\right) \neq 0$ and $V \notin \mathcal{S}\left(\mathcal{H}_{1}^{+}\right)$.

Then $V \notin \mathcal{S}(\mathcal{H})$ and $i<n$. From the argument of Case 1 of Theorem 2.5 and the assumption that $\Delta\left(r_{2}, \mu_{2}\right)>\tilde{I}\left(M_{L_{1}}, \partial \mathcal{N}\left(L_{1}\right)\right)=\tilde{I}(\mathcal{H})$, the required isotopy of $L_{2}$ in $V$ exists.

Case 2. $H_{2}\left(V_{L_{2}}, \partial V\right) \neq 0$ and $V \in \mathcal{S}\left(\mathcal{H}_{1}^{+}\right)$.

By the argument of Case 3 of Theorem 2.5, this is a contradiction.

Case 3. $H_{2}\left(V_{L_{2}}, \partial V\right)=0$.

Then, by the argument of Case 4 of Theorem 2.5, $V \in \mathcal{S}\left(\mathcal{H}_{1}^{+}\right)$and $L_{2}$ is a core of $V$. Moreover, the winding number of a suture in $V$ is $\Delta\left(r_{2}, \mu_{2}\right)$. We must show that $L_{1}$ also lies in $V$. If not, then $V \in \mathcal{S}(\mathcal{H})$ and so $\Delta\left(r_{2}, \mu_{2}\right) \leq w(\mathcal{H}) \leq$ $\tilde{I}\left(M_{L_{1}}, \partial \mathcal{N}\left(L_{1}\right)\right)$, which is a contradiction. This proves the claim.

Let $V$ be the solid torus of the claim. If $H_{2}\left(V_{L_{1}}, \partial V\right) \neq 0$, then $L_{1}$ is homotopically trivial in $V$ and hence $M$. This contradicts Theorem A.9. Hence, $H_{2}\left(V_{L_{1}}, \partial V\right)=0$. Therefore, $V_{L_{1}}$ is a rational homology cobordism, and hence a collar on $\partial \mathcal{N}\left(L_{1}\right)$, by assumption. Thus, $L_{1}$ is also a core of $V$. It is then straightforward to construct the required isotopy of $M$ which takes $L_{2}$ to $L_{1}$. This isotopy must take the slope $r_{2}$ on $\partial \mathcal{N}\left(L_{2}\right)$ to $r_{1}$ on $\partial \mathcal{N}\left(L_{1}\right)$, by Theorem A.9.

Thus, in a certain sense, the surgery curve $L_{1}$ is 'intrinsic' in $M$. There are other versions of Theorem 4.6. For example, there is a version which deals with the case where $\partial M$ is a (possibly empty) collection of tori and Dehn surgery along each $L_{i}$ reduces the Thurston norm of a given class in $H_{2}(M, \partial M)$. Using Theorem 4.6, we can prove the following theorem. 
Corollary 4.7. Let $N$ be a compact connected orientable irreducible 3-manifold with boundary. Let $P$ be a toral component of $\partial N$. Suppose that $H_{2}(N, \partial N-P) \neq$ 0 , and that the only tori in $N$ which are cobordant to $P$ by a rational homology cobordism are parallel to $P$. Let $r, \alpha$ and $\alpha_{1}$ be slopes on $P$. Assume that $\Delta(r, \alpha)>$ $\tilde{I}(N, P)$. Suppose that $N(r)$ is reducible. Then any homeomorphism from $N(\alpha)$ to $N\left(\alpha_{1}\right)$ is isotopic to a homeomorphism which takes $N$ to $N, r$ to $r$ and $\alpha$ to $\alpha_{1}$.

Thus, in both the category of homeomorphisms and the category of orientationpreserving homeomorphsims, the core of the surgery solid torus in $N(\alpha)$ is determined by its complement.

Proof. Let $(N, \gamma)$ be a taut sutured manifold structure, which exists by Theorem 2.1. By Theorem A.9, $N(\alpha)$ is irreducible. Assume now that $h: N(\alpha) \rightarrow N\left(\alpha_{1}\right)$ is a homeomorphism. Note that this implies that $\alpha_{1} \neq r$, since $N(r)$ is reducible. Let $L_{1}$ (respectively, $L$ ) be the core of the surgery solid torus in $N\left(\alpha_{1}\right)$ (respectively, $N(\alpha)$ ). Let $L_{2}$ be $h(L)$ in $N\left(\alpha_{1}\right)$. Let $r_{2}$ and $\mu_{2}$ be $h(r)$ and $h(\alpha)$, respectively, in $\partial \mathcal{N}\left(L_{2}\right)$. Note that $\Delta\left(\mu_{2}, r_{2}\right)=\Delta(\alpha, r)>\tilde{I}(N, P)$. Now apply Theorem 4.6 (with $M, \mu_{1}$ and $r_{1}$ of Theorem 4.6 being $N\left(\alpha_{1}\right), \alpha_{1}$ and $r$ ) to deduce that there is an isotopy of $N\left(\alpha_{1}\right)$ which takes $L_{2}$ to $L_{1}$, and $r_{2}$ to $r_{1}$. Hence, $h$ is isotopic to a homeomorphism which takes $N$ to $N, r$ to $r$ and $\alpha$ to $\alpha_{1}$.

Theorem 4.6 also implies Theorem 4.1.

Proof of Theorem 4.1. Define $C\left(V^{1}, K_{1}\right)$ as follows:

$$
C\left(V^{1}, K_{1}\right)= \begin{cases}\max \left\{\tilde{I}\left(V_{K_{1}}^{1}, \partial \mathcal{N}\left(K_{1}\right)\right), I\left(V^{1}\right)\right\}, & \text { if } V^{1} \text { is irreducible } \\ \tilde{I}\left(V_{K_{1}}^{1}, \partial \mathcal{N}\left(K_{1}\right)\right), & \text { if } V^{1} \text { is reducible. }\end{cases}
$$

Assume that $h_{1}: V_{K_{2}}^{2}\left(p_{2} / q_{2}\right) \rightarrow V_{K_{1}}^{1}\left(p_{1} / q_{1}\right)$ is an orientation-preserving homeomorphism, and that $\left|q_{2}\right|>C\left(V^{1}, K_{1}\right)$. Let $M$ be $V_{K_{1}}^{1}\left(p_{1} / q_{1}\right)$, let $L_{1}$ be the core of the surgery solid torus in $V_{K_{1}}^{1}\left(p_{1} / q_{1}\right)$, and let $r_{1}$ be the slope which was meridional in $V^{1}$. Similarly, let $L_{2}$ and $r_{2}$ be the images under $h_{1}$ of the core of the surgery solid torus in $V_{K_{2}}^{2}\left(p_{2} / q_{2}\right)$ and of the slope on $\partial \mathcal{N}\left(K_{2}\right)$ which was meridional in $V^{2}$. All of the conditions of Theorem 4.6 are easy to check except the following.

Claim. $V_{K_{1}}^{1}\left(p_{1} / q_{1}\right)$ is irreducible, and $r_{1} \neq \mu_{1}$.

By Theorem 2.1 (with $M$ of 2.1 being $V_{K_{2}}^{2}$ ) and Theorem A.9 (with $N$ of A.9 being $\left.V_{K_{2}}^{2}\right), V_{K_{2}}^{2}\left(p_{2} / q_{2}\right)$ is irreducible. Hence, $V_{K_{1}}^{1}\left(p_{1} / q_{1}\right)$ is also. Now, if $r_{1}=\mu_{1}$, then $V_{K_{1}}^{1}\left(p_{1} / q_{1}\right)=V^{1}$, and so by Theorem 2.1, $V^{1}$ has a taut sutured manifold structure $\left(V^{1}, \gamma\right)$. But then Theorem 2.5 (b) (with $(M, \gamma), L$ and $r$ of 2.5 being $\left(V^{1}, \gamma\right), L_{2}$ and $\left.r_{2}\right)$ implies that $\Delta\left(r_{2}, \mu_{2}\right) \leq I\left(V^{1}\right)$. But then $\left|q_{2}\right|=\Delta\left(r_{2}, \mu_{2}\right) \leq$ $I\left(V^{1}\right) \leq C\left(V^{1}, K_{1}\right)$, which is a contradiction. This proves the claim.

Theorem 4.6 now implies that there is an orientation-preserving homeomorphism $h_{2}: V_{K_{1}}^{1}\left(p_{1} / q_{1}\right) \rightarrow V_{K_{1}}^{1}\left(p_{1} / q_{1}\right)$ which takes $L_{2}$ to $L_{1}$ and $r_{2}$ to $r_{1}$. Then $h_{2} \circ$ $h_{1}$ restricts to an orientation-preserving homeomorphism from $V_{K_{2}}^{2}$ to $V_{K_{1}}^{1}$ which takes $\infty$ and $p_{2} / q_{2}$ on $\partial \mathcal{N}\left(K_{2}\right)$ to $\infty$ and $p_{1} / q_{1}$ on $\partial \mathcal{N}\left(K_{1}\right)$. This extends to an orientation-preserving homeomorphism from $V^{2}$ to $V^{1}$ which takes $K_{2}$ to $K_{1}$, meridian to meridian, longitude to longitude, and $p_{2} / q_{2}$ to $p_{1} / q_{1}$. Hence, $p_{2} / q_{2}=$ $p_{1} / q_{1}$.

Remark 4.8. Theorem 4.1 is by no means the most general possible statement. There are the following generalisations. 

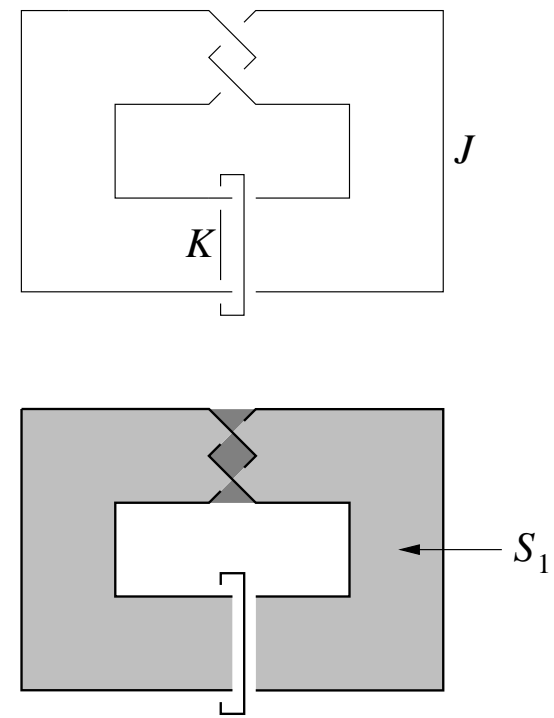

Figure 3

(i) There is a version which deals with orientation-reversing homeomorphisms. The conclusion is that, if $\left|q_{2}\right|>C\left(V^{1}, K_{1}\right)$, then

$$
V_{K_{1}}^{1}\left(p_{1} / q_{1}\right) \cong_{r} V_{K_{2}}^{2}\left(p_{2} / q_{2}\right) \Longleftrightarrow\left\{\begin{array}{c}
\left(V^{1}, K_{1}\right) \cong_{r}\left(V^{2}, K_{2}\right) \\
\text { and } \\
p_{1} / q_{1}=-p_{2} / q_{2}
\end{array}\right\},
$$

where $\cong_{r}$ denotes an orientation-reversing homeomorphism.

(ii) For $i=1$ or 2 or both, we can drop the assumption that $K_{i}$ is homotopically trivial, and assume instead that $K_{i}$ is homologically trivial and that $V^{i}$ is reducible or $\left[K_{i}\right]$ has finite order in $\pi_{1}\left(V^{i}\right)$.

(iii) We can drop the assumption that $V^{i}-K_{i}$ is atoroidal, and replace it with the hypothesis that the only tori in $V_{K_{i}}^{i}$ which are cobordant to $\partial \mathcal{N}\left(K_{i}\right)$ by a rational homology cobordism are parallel to $\partial \mathcal{N}\left(K_{i}\right)$.

Example 4.9. Let $J \cup K$ be the Whitehead link in $S^{3}$ shown in Figure 3. Let $V$ be the solid torus $S^{3}-\operatorname{int}(\mathcal{N}(J))$. It is not hard to show there are no essential tori in $S^{3}-\operatorname{int}(\mathcal{N}(J \cup K))$. Note that $K$ is homologically trivial in the solid torus $V$, and hence it is homotopically trivial.

Claim. $\tilde{I}\left(V_{K}, \partial \mathcal{N}(K)\right) \leq 2$.

We shall construct a sutured manifold sequence $\mathcal{H}$ as in Definition 4.3. The first sutured manifold is $\left(V_{K}, \emptyset\right)$. Let the first decomposing surface $S_{1}$ be the Seifert surface for $J$ shown in Figure 3 . Let $\left(N_{2}, \gamma_{2}\right)$ be the sutured manifold which results from the decomposition. Let the second decomposing surface $S_{2}$ be the obvious product disc in $N_{2}$. Let $\left(N_{3}, \gamma_{3}\right)$ be the sutured manifold which results from this decomposition. Then $N_{3}$ is a collar on $\partial \mathcal{N}(K)$, and $\gamma_{3}$ is two simple closed curves which are parallel to the meridional slope on $\partial \mathcal{N}(K)$. Thus, $\tilde{I}\left(V_{K}, \partial \mathcal{N}(K)\right) \leq$ $\tilde{I}(\mathcal{H})=2$. 
Now, if $n \in \mathbb{Z}-\{0\}$, then $V_{K}(-1 / n)$ is $S^{3}-\operatorname{int}\left(\mathcal{N}\left(T_{n}\right)\right)$, where $T_{n}$ is a twist knot. Theorem 4.4 (with $N=V_{K}$ and $r=\infty$ and $\alpha=-1 / n$ ) implies that

$$
I\left(S^{3}-\operatorname{int}\left(\mathcal{N}\left(T_{n}\right)\right)\right) \begin{cases}=|n|, & \text { if }|n| \geq 2 \\ \leq 2, & \text { if }|n|=1 .\end{cases}
$$

This has the obvious corollary that the complements of $T_{n}$ and $T_{m}$ are not homeomorphic if $|n| \neq|m|$ and $|m|>2$. Hence, in this case, $T_{n}$ and $T_{m}$ are distinct knots.

Theorem 4.6 states that the core $L_{1}$ of the surgery solid torus in $S^{3}-\operatorname{int}\left(\mathcal{N}\left(T_{n}\right)\right)$ is an 'intrinsic surgery curve', in the following sense. Suppose that $L_{2}$ is a curve in $S^{3}-\operatorname{int}\left(\mathcal{N}\left(T_{n}\right)\right)$ which does not lie in a 3-ball. Suppose also that $S^{3}-\operatorname{int}\left(\mathcal{N}\left(T_{n} \cup L_{2}\right)\right)$ is atoroidal, and that $L_{2}$ and $T_{n}$ have zero linking number. If Dehn surgery along $L_{2}$ in $S^{3}-\operatorname{int}\left(\mathcal{N}\left(T_{n}\right)\right)$ with coefficient $p / q(|q|>2)$ yields a solid torus or a reducible manifold, then $L_{2}$ is ambient isotopic to $L_{1}$ in $S^{3}-\operatorname{int}\left(\mathcal{N}\left(T_{n}\right)\right)$, and $p / q=1 / n$. Note that the restriction on $|q|$ is vital. For, it is possible to unknot $T_{n}$ by changing a crossing in the 'clasp'. This crossing change is effected by Dehn surgery with coefficient -1 about an unknotted curve.

\section{LONGITUDES}

One of the main subjects of this paper has been the set of slopes $r$ such that $M_{L}(r)$ is a reducible manifold, and in particular, the integer $\Delta(r, \mu)$ has been studied. In this section, we define the notion of a longitudinal slope $\lambda$ on $\partial \mathcal{N}(L)$. When $L$ is homologically trivial in $M$, this coincides with the standard terminology. What can be said about $\Delta(r, \lambda)$ ? We consider the Euler characteristic of a compact connected oriented surface $F$ properly embedded in $M-\operatorname{int}(\mathcal{N}(L))$, such that $F \cap \partial \mathcal{N}(L)$ is a non-empty collection of coherently oriented curves of slope $\lambda$. Thus, if $L$ is homologically trivial in $M$, such a surface arises from a spanning surface for $L$. We demonstrate that if Dehn surgery on $L$ with slope $r$ yields a reducible manifold, then, under most circumstances, $\Delta(r, \lambda)$ is bounded by the modulus of the Euler characteristic of the surface $F$.

Note that, in general, there is no upper bound on $\Delta(r, \lambda)$ which depends only on $M$. For, when $L$ is the $(p, q)$ cable of a homologically trivial knot in $M$ and $|q|>1$, then $p q$ surgery along $L$ yields a reducible manifold. Therefore, by a suitable choice of $L, \Delta(r, \lambda)=|p q|$ can be arbitrarily large.

Definition 5.1. Let $L$ be a knot in a compact orientable 3-manifold $M$, possibly with boundary. A slope $\lambda$ on $\partial \mathcal{N}(L)$ is a longitude if there is a compact connected oriented surface $F$ properly embedded in $M_{L}$ such that $F \cap \partial \mathcal{N}(L)$ is a non-empty collection of coherently oriented curves with slope $\lambda$.

Lemma 5.2. Let $L$ be a knot in a compact orientable 3-manifold $M$, possibly with boundary. Then there is at least one longitudinal slope on $\partial \mathcal{N}(L)$.

Proof. Let $N$ be the manifold obtained by attaching a ball or handlebody to each boundary component of $M$. Since $N$ is closed, the map $i_{*}: H_{1}(\partial \mathcal{N}(L)) \rightarrow H_{1}\left(N_{L}\right)$ has non-trivial kernel. Thus, there is an oriented (possibly disconnected) surface $F$ properly embedded in $N_{L}$ such that $\partial F \cap \partial \mathcal{N}(L)$ is non-trivial in $H_{1}(\partial \mathcal{N}(L))$. By attaching discs to curves of $F \cap \partial \mathcal{N}(L)$ which are inessential in $\partial \mathcal{N}(L)$, and then attaching annuli to curves of $F \cap \partial \mathcal{N}(L)$ which are incoherently oriented in $\partial \mathcal{N}(L)$, we may assume that the curves of $F \cap \partial \mathcal{N}(L)$ are essential and coherently oriented 
in $\partial \mathcal{N}(L)$. Some component $F_{i}$ of $F$ has $F_{i} \cap \partial \mathcal{N}(L)$ non-trivial in $H_{1}(\partial \mathcal{N}(L))$. Each curve of $F_{i} \cap \partial \mathcal{N}(L)$ has the required longitudinal slope, since $F_{i} \cap M_{L}$ is the required surface.

There may be more than one longitudinal slope on $\partial \mathcal{N}(L)$. For example, when $M_{L}$ is $S^{1} \times S^{1} \times I$, then every slope is a longitude.

Theorem 5.3. Let $M$ be a compact connected orientable irreducible 3-manifold such that $\partial M$ is a (possibly empty) collection of tori. Let $L$ be a knot in $M$ which does not lie in a 3-ball. Let $\lambda$ be a longitudinal slope on $\partial \mathcal{N}(L)$ and let $r$ be another slope. Let $F$ be a compact connected oriented surface properly embedded in $M_{L}$, with $\partial F \cap \partial \mathcal{N}(L)$ a non-empty collection of coherently oriented curves with slope $\lambda$. Let $z$ be a non-trivial element of $H_{2}\left(M_{L}, \partial M\right)$. Let $z_{\mu}$ and $z_{r}$ be the elements of $H_{2}(M, \partial M)$ and $H_{2}\left(M_{L}(r), \partial M_{L}(r)\right)$ to which $z$ is sent by the maps induced by inclusion. Suppose that the only tori in $M_{L}$ which are cobordant to $\partial \mathcal{N}(L)$ by a rational homology cobordism are parallel to $\partial \mathcal{N}(L)$. If $M_{L}(r)$ is reducible, or the core of the surgery solid torus in $M_{L}(r)$ has finite order in $\pi_{1}\left(M_{L}(r)\right)$, or the Thurston norm of $z_{r}$ is less than that of $z_{\mu}$ or $z$, then

$$
\Delta(r, \lambda) \leq \frac{-\chi(F)}{|F \cap \partial \mathcal{N}(L)|} .
$$

Proof. Let $F^{\prime}$ be the surface in $M_{L}(\lambda)$ which is obtained from $F$ by adding a meridian disc to each curve of $\partial F \cap \partial \mathcal{N}(L)$. Then $\chi\left(F^{\prime}\right)=\chi(F)+|F \cap \partial \mathcal{N}(L)|$. Let $L^{\prime}$ be the core of the surgery solid torus in $M_{L}(\lambda)$. Then, $L^{\prime}$ has intersection number $|F \cap \partial \mathcal{N}(L)|$ with $F^{\prime}$. Now, $\left(M_{L}, \emptyset\right)$ is a taut sutured manifold. If $F^{\prime}$ is a sphere, then, as $F^{\prime}$ is non-separating, $M_{L}(\lambda)$ is reducible. If $F^{\prime}$ is a disc which is not a compression disc for $\partial M_{L}(\lambda)$, then $M_{L}(\lambda)$ is again reducible. If $F^{\prime}$ is a compression disc for $\partial M_{L}(\lambda)$, then $M_{L}(\lambda)$ is reducible or a solid torus. However, in the latter case, $L^{\prime}$ has non-zero winding number and so $H_{2}\left(M_{L}, \partial M\right)=0$, which is a contradiction. Thus, if $F^{\prime}$ is a sphere or disc, then $M_{L}(\lambda)$ is reducible. This is a contradiction, by Corollary A.8 (with $N=M_{L}$ ), and so $F^{\prime}$ is not a sphere or disc. If $\Delta(r, \lambda) \neq 1$, apply Theorem A.10 (b) (with $M, \gamma, L$ and $F$ of Theorem A.10 being $M_{L}(\lambda), \emptyset, L^{\prime}$ and $F^{\prime}$ ) to deduce that we may ambient isotope $L^{\prime}$ in $M_{L}(\lambda)$ so that

$$
\left|L^{\prime} \cap F^{\prime}\right| \leq \frac{-\chi\left(F^{\prime}\right)}{(\Delta(r, \lambda)-1)} .
$$

However, $\left|L^{\prime} \cap F^{\prime}\right| \geq|F \cap \partial \mathcal{N}(L)|$, since the latter is the intersection number of $L^{\prime}$ and $F^{\prime}$. Hence,

$$
|F \cap \partial \mathcal{N}(L)|(\Delta(r, \lambda)-1) \leq-\chi\left(F^{\prime}\right)=-\chi(F)-|F \cap \partial \mathcal{N}(L)| .
$$

Note that this inequality is automatically satisfied if $\Delta(r, \lambda)=1$, since $F^{\prime}$ is not a sphere or disc. Therefore,

$$
\Delta(r, \lambda) \leq \frac{-\chi(F)}{|F \cap \partial \mathcal{N}(L)|}
$$

The above theorem has a number of generalisations and variations. For example, one can state a version where $M$ is an arbitrary compact connected orientable 3manifold with a taut sutured manifold structure. However, Theorem 5.3 has the advantage of simplicity and clarity, and any alternatives are left to the reader. 


\section{APPENDIX: NEW VERSIONS OF OLD RESULTS}

In this section, we state and prove some stronger versions of well-known results regarding sutured manifolds.

In [1], Gabai introduced a version of the following definition.

Definition A.1. An I-cobordism between closed orientable, possibly disconnected, surfaces $P_{1}$ and $P_{2}$ is a compact orientable 3-manifold $H$, such that $\partial H=$ $P_{1} \cup P_{2}$ and such that, for each $j$, the maps $i_{*}: H_{1}\left(P_{j}\right) \rightarrow H_{1}(H)$ induced by inclusion are injective.

In [11], Scharlemann introduced the following definition.

Definition A.2. Suppose that $H$ is a compact orientable 3-manifold and that $P \subset \partial H$ is a closed, possibly disconnected, surface. Then $H$ is a $\mathbf{J}$-cobordism on $P$ if $H_{2}(H, \partial H-P)=0$.

Here, we recall the following well-known definition.

Definition A.3. A rational homology cobordism between closed orientable, possibly disconnected, surfaces $P_{1}$ and $P_{2}$ is a compact orientable 3-manifold $H$, such that $\partial H=P_{1} \cup P_{2}$ and such that, for each $i$, the inclusion maps of $P_{i}$ into $H$ induce isomorphisms between their rational homology groups.

These definitions are all related, as is demonstrated in the following lemma.

Lemma A.4. Suppose that $H$ is a compact orientable 3-manifold and that $\partial H=$ $P_{1} \cup P_{2}$, where each $P_{i}$ is a closed orientable surface.

(a) If $H$ is a rational homology cobordism between $P_{1}$ and $P_{2}$, then it is a $J$ cobordism on $P_{i}$ for each $i$.

(b) If each component of $H$ contains at least one component of $P_{1}$ and at least one component of $P_{2}$, and $H$ is a J-cobordism on $P_{i}$ for each $i$, then $H$ is a rational homology cobordism between $P_{1}$ and $P_{2}$.

(c) If $H$ is a J-cobordism on $P_{i}$ for each $i$, then it is an I-cobordism between $P_{1}$ and $P_{2}$.

(d) If $P_{1}$ is a single torus and $P_{2}$ is non-empty, and $H$ is a connected irreducible $J$-cobordism on $P_{1}$, then $H$ is a rational homology cobordism between $P_{1}$ and $P_{2}$.

Proof. (a) It follows trivially from the rational homology exact sequence associated with the pair $\left(H, P_{i}\right)$ that $H_{2}\left(H, P_{i} ; \mathbb{Q}\right)=0$, for each $i$. Since $H_{2}\left(H, P_{i}\right)$ is free, this implies that $H_{2}\left(H, P_{i}\right)$ is trivial.

(b) It suffices to show that $H_{j}\left(H, P_{i} ; \mathbb{Q}\right)=0$ for each $j$ and $i$. This is true for $j=0$ and 2 by assumption. It is therefore true for $j=1$ and 3 , by duality. It is trivially true for all $j>3$.

(c) follows from the homology exact sequence associated with the pair $\left(H, P_{i}\right)$.

(d) It suffices to show that $H$ is a J-cobordism on $P_{2}$, by (b). To do this, it suffices to prove that $H_{2}\left(H, P_{1} ; \mathbb{Q}\right)=0$. This follows from Claims 1 and 2 below.

Claim 1. The map $i_{*}: H_{1}\left(P_{1} ; \mathbb{Q}\right) \rightarrow H_{1}(H ; \mathbb{Q})$ induced by inclusion is injective.

If $\partial H$ contains a sphere component, then this bounds a ball in $H$, which is a contradiction. By Lemma 2.2 of [11], genus $\left(P_{2}\right) \leq \operatorname{genus}\left(P_{1}\right)=1$. Hence, $P_{2}$ is a single torus. By the rational homology exact sequence associated with the pair $\left(H, P_{2}\right)$, the map $i_{*}: H_{1}\left(P_{2} ; \mathbb{Q}\right) \rightarrow H_{1}(H ; \mathbb{Q})$ induced by inclusion is injective. Lemma 1.5 of $[1]$ implies that the same is true of the map $i_{*}: H_{1}\left(P_{1} ; \mathbb{Q}\right) \rightarrow H_{1}(H ; \mathbb{Q})$. 
Claim 2. The map $i_{*}: H_{2}\left(P_{1} ; \mathbb{Q}\right) \rightarrow H_{2}(H ; \mathbb{Q})$ induced by inclusion is surjective.

Now, $H_{2}\left(H, P_{2} ; \mathbb{Q}\right)=H_{3}\left(H, P_{2} ; \mathbb{Q}\right)=0$ and so $H_{2}(H ; \mathbb{Q}) \cong H_{2}\left(P_{2} ; \mathbb{Q}\right) \cong \mathbb{Q}$. Also, $H_{3}\left(H, P_{1} ; \mathbb{Q}\right)=0$ and so the map $i_{*}: H_{2}\left(P_{1} ; \mathbb{Q}\right) \rightarrow H_{2}(H ; \mathbb{Q})$ induced by inclusion is injective. But $H_{2}\left(P_{1} ; \mathbb{Q}\right) \cong \mathbb{Q}$, and an injective linear map from $\mathbb{Q}$ to $\mathbb{Q}$ is a surjection.

Examples A.5. The following examples demonstrate that the notions of an Icobordism, J-cobordism and rational homology cobordism are not equivalent.

(i) Let $H$ be the connected sum of $S^{1} \times S^{1} \times I$ with a closed 3-manifold with non-trivial first rational homology. Then $H$ is an I-cobordism between its two boundary components but not a J-cobordism on either boundary component.

(ii) Let $H$ be a solid torus with the interior of an embedded 3-ball removed. Then $H$ is a J-cobordism on the toral boundary component, but is not a rational homology cobordism between its boundary components.

The point of introducing all these definitions is that, throughout much of sutured manifold theory, hypotheses about I-cobordisms in a manifold have been needed. However, these can each be weakened to hypotheses about rational homology cobordisms. For example, one can prove the following result, which should be compared with Theorem 1.7 of [1].

Theorem A.6. Let $N$ be a compact connected orientable irreducible 3-manifold with $\partial N$ a non-empty collection of tori. Let $S$ be a non-empty taut surface in $N$. Suppose that no non-empty collection of curves in $\partial S$ is homologically trivial in $\partial N$, and that no component of $S$ is closed and separating. Let $P$ be a component of $\partial N$ disjoint from $S$. Suppose that the only tori in $N-S$ which are cobordant to $P$ by a rational homology cobordism are parallel to $P$. Then, for all slopes $\alpha$ on $P$, except possibly one slope, the following statements hold in the manifold $N(\alpha)$ :

(i) $S$ is taut in $N(\alpha)$, and

(ii) $N(\alpha)$ is irreducible, and

(iii) the core of the surgery solid torus has infinite order in $\pi_{1}(N(\alpha))$.

Proof. This is proved in the same way as Theorem 5.1 of [9]. Note first that $N$ is not a solid torus. For then $S$ would be disjoint from $\partial N$, and $H_{2}(N)$ would be trivial. Hence, each component of $S$ would be closed and separating, contrary to assumption. Thus, as $N$ is also irreducible, $N$ has a taut sutured manifold structure $(N, \emptyset)$, with $R_{-}=\partial N$ and $R_{+}=\emptyset$. In the proof of Theorem 5.1 of [9], a taut sutured manifold sequence

$$
\left(M_{0}, \gamma_{0}\right) \stackrel{S_{1}}{\longrightarrow}\left(M_{1}, \gamma_{1}\right) \stackrel{S_{2}}{\longrightarrow} \ldots \stackrel{S_{n}}{\longrightarrow}\left(M_{n}, \gamma_{n}\right)
$$

is constructed, such that

(a) $\left(M_{0}, \gamma_{0}\right)=(N, \emptyset)$,

(b) each $S_{i}$ is either a conditioned surface, a product disc, or a non-trivial product annulus,

(c) no closed component of any $S_{i}$ separates,

(d) each $S_{i}$ is disjoint from $P$, and

(e) $H_{2}\left(M_{n}, \partial M_{n}-P\right)=0$.

Let $H$ be the component of $M_{n}$ containing $P$. Then $H$ is a J-cobordism on $P$. $H$ is irreducible, since $\left(M_{n}, \gamma_{n}\right)$ is taut. Also, $\partial H-P$ is non-empty, since at least one decomposition has taken place. Thus, $H$ is a rational homology cobordism, by 
Lemma A.4 (d). Hence, $H$ is homeomorphic to $P \times I$. It is then argued in Theorem 5.1 of [9] that for all slopes $\alpha$ on $P$, except possibly one slope, $\left(M_{i}(\alpha), \gamma_{i}-P\right)$ is taut for all $i$. This implies (ii) immediately. Corollary 3.3 of [9] and the fact that no component of $\partial S_{i}$ bounds a disc in $R_{ \pm}\left(\gamma_{i-1}\right)$ for any $i$ imply that each decomposing surface $S_{i}$ is taut in $M_{i-1}(\alpha)$. This gives (i). By the argument of Lemma 6.1 of [3], the map $i_{*}: \pi_{1}(H(\alpha)) \rightarrow \pi_{1}(N(\alpha))$ induced by inclusion is an injection. Since $\pi_{1}(H(\alpha))$ is a copy of $\mathbb{Z}$ generated by the core of the surgery solid torus, (iii) follows immediately.

The following result is implicit in much of the previous work on sutured manifolds. There are stronger versions of this result, but we will not need them.

Lemma A.7. Let $N$ be a compact orientable 3-manifold with $\partial N$ a collection of tori. Let $S$ be an oriented surface properly embedded in $N$, such that no component of $\partial S$ bounds a disc in $\partial N$. Then $S$ is norm minimising in $[S, \partial S] \in H_{2}(N, \partial N)$ if and only if it is norm minimising in $[S, \partial S] \in H_{2}(N, \mathcal{N}(\partial S))$.

Proof. It is clear that if $S$ is norm minimising in $[S, \partial S] \in H_{2}(N, \partial N)$, then it is norm minimising in $[S, \partial S] \in H_{2}(N, \mathcal{N}(\partial S))$. For the converse, assume that $S$ is norm minimising in $[S, \partial S] \in H_{2}(N, \mathcal{N}(\partial S))$, and that $S^{\prime}$ is a norm minimising representative of $[S, \partial S] \in H_{2}(N, \partial N)$. By attaching discs and annuli to $S^{\prime}$, we may assume that no non-empty collection of curves in $\partial S^{\prime}$ is homologically trivial in $\partial N$. We may therefore assume that $\partial S^{\prime}$ lies in $\mathcal{N}(\partial S)$. Now, $H_{2}(\partial N, \mathcal{N}(\partial S))$ is generated by oriented annuli and tori. By the homology exact sequence associated with the triple $(N, \partial N, \mathcal{N}(\partial S))$, we may find a union $F$ of oriented annuli and tori disjoint from $S^{\prime}$ and from each other, such that $\left[S^{\prime}, \partial S^{\prime}\right]+[F, \partial F]=[S, \partial S] \in$ $H_{2}(N, \mathcal{N}(\partial S))$. Hence, $\chi_{-}\left(S^{\prime}\right)=\chi_{-}(S)$.

The following result is a version of Theorem 5.1 of [9].

Corollary A.8. Let $N$ be a compact connected orientable irreducible 3-manifold with $\partial N$ a non-empty collection of tori. Let $P$ be a component of $\partial N$. Suppose that the only tori in $N$ which are cobordant to $P$ by a rational homology cobordism are parallel to $P$. Let $z$ be a non-trivial element of $H_{2}(N, \partial N-P)$. For each slope $\alpha$ on $P$, let $z_{\alpha} \in H_{2}(N(\alpha), \partial N(\alpha))$ be the image of $z$ under the map induced by inclusion. Then for all slopes $\alpha$ on $P$, except possibly one slope, the following statements hold in the manifold $N(\alpha)$ :

(i) The Thurston norms of $z$ and $z_{\alpha}$ are equal, and

(ii) $N(\alpha)$ is irreducible, and

(iii) the core of the surgery solid torus has infinite order in $\pi_{1}(N(\alpha))$.

Proof. Let $S$ be a taut representative of $z$. We may assume that no non-empty collection of curves in $\partial S$ is homologically trivial in $\partial N$, and that $S$ has no closed separating components. By Lemma A.7, $S$ is norm minimising in $[S, \partial S] \in$ $H_{2}(N(\alpha), \mathcal{N}(\partial S))$ if and only if the Thurston norms of $z$ and $z_{\alpha}$ are equal. Now apply Theorem A.6.

There is also the following version of Theorem A.6 for an arbitrary taut sutured manifold $(N, \gamma)$.

Theorem A.9. Let $(N, \gamma)$ be a connected taut sutured manifold with $\partial N$ containing a toral component $P$. Suppose that the only tori in $N$ which are cobordant to $P$ by a rational homology cobordism are parallel to $P$. Suppose that $H_{2}(N, \partial N-P)$ 
is non-trivial. Then, for all slopes $\alpha$ on $P$, except possibly one slope, the following hold:

(i) $(N(\alpha), \gamma-P)$ is taut, and

(ii) the core of the surgery solid torus has infinite order in $\pi_{1}(N(\alpha))$.

We also note that Theorem 1.4 of [6] can be phrased in terms of rational homology cobordisms instead of I-cobordisms. The following is the new version. It has a number of other minor improvements, but its proof remains virtually unchanged. It is the central tool in proving the main results of this paper.

Theorem A.10. Let $M$ be a compact connected orientable 3-manifold, possibly with boundary. Let $L$ be a knot in $M$. Suppose that $\left(M_{L}, \gamma\right)$ is a taut sutured manifold with $\gamma \cap \partial \mathcal{N}(L)=\emptyset$. Let $\mu$ be the meridional slope on $\partial \mathcal{N}(L)$. Let $r$ be a slope on $\partial \mathcal{N}(L)$ such that $\Delta(r, \mu)>1$. Suppose that $z$ is a non-trivial element of $H_{2}\left(M_{L}, \partial M\right)$. Let $F$ be a properly embedded orientable surface in $M$ with components $F_{1}, \ldots, F_{|F|}$. Assume that no component is a sphere or disc disjoint from $\gamma$.

(a) Suppose that the only tori in $M_{L}$ which are cobordant to $\partial \mathcal{N}(L)$ by a rational homology cobordism are parallel to $\partial \mathcal{N}(L)$. If $\left(M_{L}(r), \gamma\right)$ is not taut or the core of the surgery solid torus in $M_{L}(r)$ has finite order in $\pi_{1}\left(M_{L}(r)\right)$, then there is an ambient isotopy of $L$ in $M$ so that, after the isotopy, for each $i$, we have

$$
\left|L \cap F_{i}\right| \leq \frac{-2 \chi\left(F_{i}\right)+\left|F_{i} \cap \gamma\right|}{2(\Delta(r, \mu)-1)} .
$$

(b) Suppose that $\partial M$ is a (possibly empty) collection of tori and that $\gamma=\emptyset$. Let $S$ be some taut conditioned surface properly embedded in $M_{L}$, which is disjoint from $\partial \mathcal{N}(L)$, which has no closed separating component, and such that $[S, \partial S]=$ $z \in H_{2}\left(M_{L}, \partial M\right)$. Suppose that any torus in $M-\operatorname{int}(\mathcal{N}(L \cup S))$ which is cobordant to $\partial \mathcal{N}(L)$ by a rational homology cobordism is parallel to $\partial \mathcal{N}(L)$. If $S$ is not taut in $M_{L}(r)$, or $\left(M_{L}(r), \gamma\right)$ is not taut, or the core of the surgery solid torus in $M_{L}(r)$ has finite order in $\pi_{1}\left(M_{L}(r)\right)$, then there is an ambient isotopy of $L$ in $M$ so that, after the isotopy, for each $i$, we have

$$
\left|L \cap F_{i}\right| \leq \frac{-2 \chi\left(F_{i}\right)+\left|F_{i} \cap \gamma\right|}{2(\Delta(r, \mu)-1)} .
$$

The following results (Lemmas A.11 to A.20) help us to eliminate the assumptions about rational homology cobordisms. Many of them are trivial and their proofs are only sketched. Many are implicit in the arguments of [1].

Lemma A.11. Let $T$ be a separating torus properly embedded in a compact connected orientable 3-manifold $Q$. Let $X$ and $Y$ be the closure of the components of $Q-T$. Suppose that $T$ is incompressible in $X$ and that each 2-sphere in $X$ bounds a rational homology 3-ball in $X$. Suppose that $Y$ is a rational homology solid torus, with boundary T. Suppose that there is a 2-sphere in $Q$ which does not bound a rational homology 3-ball. Then $T$ compresses in $Y$. Moreover, if $\alpha_{1}$ is the slope on $T$ which is trivial in $H_{1}(Y ; \mathbb{Q})$, then there is a 2-sphere in $X\left(\alpha_{1}\right)$ which does not bound a rational homology 3-ball.

Proof. Suppose that $T$ is incompressible in $Y$. Then an innermost curve argument establishes that there is a 2-sphere in $X$ or $Y$ which does not bound a rational homology 3 -ball in $Q$. By assumption, it must lie in $Y$, and so $Y \cong V \# W$, where 
$V$ has boundary $T$ and $W$ is a closed manifold which is not a rational homology 3 -sphere. However, $H_{1}(V ; \mathbb{Q})$ is non-trivial, since the rank of the image of $H_{1}(T ; \mathbb{Q})$ in $H_{1}(V ; \mathbb{Q})$ under the map induced by inclusion is one. Therefore, $Y$ cannot be a rational homology solid torus, which is a contradiction. Thus, $T$ compresses in $Y$.

We deduce now that $Y \cong D^{2} \times S^{1} \# W$ for some closed 3-manifold $W$, which must be a rational homology 3 -sphere. Thus, $Q \cong X\left(\alpha_{1}\right) \# W$. Using another innermost curve argument, we deduce that there is a 2-sphere in $X\left(\alpha_{1}\right)$ which does not bound a rational homology 3-ball.

Lemma A.12. Let $T$ be a separating torus properly embedded in a compact connected orientable 3-manifold $Q$. Let $X$ and $Y$ be the closure of the components of $Q-T$. Suppose that $X$ is irreducible and that $T$ is incompressible in $X$. If $Q$ is reducible, then $Y$ is either a solid torus or reducible.

Proof. This is a simpler version of Lemma A.11.

Lemma A.13. Let $T$ be a closed separating surface properly embedded in a compact connected orientable 3-manifold $Q$. Let $X$ and $Y$ be the closure of the components of $Q-T$. Let $S$ be a surface in $X$ which is incompressible in $X$. If $S$ is compressible in $Q$, then $T$ is compressible in $Q$.

Proof. This is a simple innermost curve argument.

Lemma A.14. Let $N$ be a compact orientable 3-manifold. Let $H$ be a rational homology cobordism in $N$ between a toral component $P$ of $\partial N$ and a properly embedded torus $T$. Let $F$ be a (possibly empty) subset of $\partial N-P$. Then the map $i_{*}: H_{2}(N-\operatorname{int}(H), F) \rightarrow H_{2}(N, F)$ induced by inclusion is an isomorphism.

Proof. For any $i, H_{i}(N, N-\operatorname{int}(H)) \cong H_{i}(H, T)$, by excision. Now, $H_{2}(H, T ; \mathbb{Q})$ and $H_{3}(H, T ; \mathbb{Q})$ are trivial, by assumption, and so $H_{2}(H, T)$ and $H_{3}(H, T)$ are trivial, since they are free. The lemma now follows from the homology exact sequence associated with the triple $(N, N-\operatorname{int}(H), F)$.

Lemma A.15. Let $N$ be a compact connected orientable irreducible 3-manifold. Let $H$ be a rational homology cobordism in $N$ between a toral component $P$ of $\partial N$ and a properly embedded torus $T$. Let $F$ be a (possibly empty) subset of $\partial N-P$. If $H_{2}(N, F)$ is non-trivial or $\partial N \neq P$, then $T$ is incompressible in $N$.

Proof. Suppose that $D$ is a compression disc for $T$. Then, $D \cap H=\partial D$, since $H$ is a rational homology cobordism. The sphere obtained by compressing $T$ bounds a ball in $N$. One deduces that $N-\operatorname{int}(H)$ is homeomorphic to a solid torus. Hence, $\partial N=P$, and so $F=\emptyset$. Therefore, $H_{2}(N-\operatorname{int}(H), F)$ is trivial. Lemma A.14 then implies that $H_{2}(N, F)$ is trivial, which is a contradiction.

Lemma A.16. Let $S$ be an oriented properly embedded surface in a compact orientable 3-manifold $N$. Let $T$ be an incompressible torus properly embedded in $N$. Suppose that $S$ is homologous rel $\partial S$ to a surface disjoint from $T$. Then, $S$ is homologous rel $\partial S$ to a taut surface disjoint from $T$.

Proof. We may assume that $S$ is taut in $N$. By Lemma 1.3 (d) of [9], we may modify $S$ by 2 -surgery to a surface $S_{1}$ so that, for any collection $k T$ of $k$ parallel identically oriented copies of $T$, the double-curve sum $S_{2}$ of $S_{1}$ and $k T$ satisfies $\chi_{-}\left(S_{2}\right)=\chi_{-}(S)+\chi_{-}(k T)=\chi_{-}(S)$. If $k$ is large enough, then, by Lemma 3.2 (b) of [9], $S_{2}$ is ambient isotopic (via an isotopy fixed on $\partial N$ ) to a surface $S_{3}$ disjoint from 
$T$. Let $S_{4}$ be the union of $S_{3}$ with $k$ parallel copies of $T$ oriented in the opposite way from previously. Then, $S_{4}$ is disjoint from $T$ and $\left[S_{4}, \partial S_{4}\right]=[S, \partial S] \in H_{2}(N, \partial S)$. Now, compress $S_{4}$ as far as possible in $N$. Since $T$ is incompressible, we may assume that the compression discs are disjoint from $T$. The resulting surface is the required taut representative.

Lemma A.17. Let $S$ be an oriented properly embedded surface in a compact connected orientable irreducible 3-manifold $N$. Let $P$ be a toral boundary component of $N$ disjoint from $S$. Let $H$ be a rational homology cobordism in $N$ between $P$ and a properly embedded torus $T$. Then, $S$ is homologous rel $\partial S$ to a taut surface disjoint from $H$.

Proof. We may assume that $[S, \partial S] \in H_{2}(N, \partial S)$ is non-trivial. Therefore, by Lemma A.15, $T$ is incompressible in $N$. By Lemma A.14, $S$ is homologous rel $\partial S$ to a surface $S_{1}$ disjoint from $T$. Lemma A.16 implies that we may assume that $S_{1}$ is taut. Now, $H_{2}(H, T)$ is trivial, and hence each component of $S_{1}$ in $H$ is either nul-homologous or homologous to $T$. We may therefore replace these components with a number of oriented copies of $T$ without increasing the Thurston norm of the surface. The resulting surface is the required taut representative.

Lemma A.18. Let $N$ be a compact connected orientable irreducible 3-manifold. Let $\alpha$ be a slope on a toral component $P$ of $\partial N$. Suppose that $P \neq \partial N$, or that $\mathrm{H}_{2}(\mathrm{~N}, \partial \mathrm{N}-\mathrm{P})$ is non-trivial. Let $\mathrm{H}$ be a rational homology cobordism in $\mathrm{N}$ between $P$ and a properly embedded torus $T$. Let $S$ be a taut surface in $N$ disjoint from $H$. Suppose that $S$ is not taut in $N(\alpha)$. Then $T$ compresses in $H(\alpha)$. Moreover, if $X=N-\operatorname{int}(H)$ and $\alpha_{1}$ is the slope on $T$ which is trivial in $H_{1}(H(\alpha) ; \mathbb{Q})$, then $S$ is not taut in $X\left(\alpha_{1}\right)$.

Proof. Lemma A.15 (with $F=\partial N-P$ ) implies that $T$ does not compress in $X$. Suppose that $T$ does not compress in $H(\alpha)$. Then Lemma A.16 (with $N$ of A.16 being $N(\alpha)$ ) implies that there is a taut representative $S_{1}$ of $[S, \partial S] \in$ $H_{2}(N(\alpha), \partial S)$ which is disjoint from $T$. Since $H_{2}(H(\alpha))$ is trivial, we may assume that $S_{1}$ is disjoint from $H(\alpha)$ and so lies in $N$. Then $S_{1}$ together with a number of oriented copies of $P$ represents $[S, \partial S] \in H_{2}(N, \partial S)$. This then implies that $\chi_{-}(S)=\chi_{-}\left(S_{1}\right)$. Also, Lemma A.13 (with $Q=N(\alpha)$ and $Y=H(\alpha)$ ) implies that $S$ is incompressible in $N(\alpha)$. Hence $S$ is taut in $N(\alpha)$, contrary to assumption. Thus, $T$ compresses in $H(\alpha)$. We deduce that $H(\alpha)=D^{2} \times S^{1} \# W$ for some closed 3 -manifold $W$. Thus, $N(\alpha) \cong X\left(\alpha_{1}\right) \# W$. Hence, $S$ is not taut in $X\left(\alpha_{1}\right)$.

Lemma A.19. Let $N$ be a compact connected orientable irreducible 3-manifold. Let $\alpha$ be a slope on a toral component $P$ of $\partial N$. Suppose that $P \neq \partial N$ or that $H_{2}(N, \partial N-P) \neq 0$. Let $H$ be a rational homology cobordism in $N$ between $P$ and a properly embedded torus $T$. Let $X=N-\operatorname{int}(H)$, and let $\alpha_{1}$ be the slope on $T$ which is trivial in $H_{1}(H(\alpha) ; \mathbb{Q})$. Let $L$ (respectively $L_{1}$ ) be the core of the surgery solid torus in $N(\alpha)$ (respectively $X\left(\alpha_{1}\right)$ ). If $[L]$ has finite order in $\pi_{1}(N(\alpha))$, then $T$ compresses in $H(\alpha)$ and $\left[L_{1}\right]$ has finite order in $\pi_{1}\left(X\left(\alpha_{1}\right)\right)$.

Proof. Note that $[L]$ has infinite order in $H_{1}(H(\alpha) ; \mathbb{Q})$ and hence in $\pi_{1}(H(\alpha))$. Lemma A.15 (with $F=\partial N-P$ ) implies that $T$ is incompressible in $X$. If $T$ does not compress in $H(\alpha)$, then the argument of Lemma 6.1 of [3] implies that the map $i_{*}: \pi_{1}(H(\alpha)) \rightarrow \pi_{1}(N(\alpha))$ induced by inclusion is an injection. But this implies that $[L]$ has infinite order in $\pi_{1}(N(\alpha))$, contrary to hypothesis. Hence, $T$ 
compresses in $H(\alpha)$, and so $N(\alpha) \cong X\left(\alpha_{1}\right) \# W$, where $W$ is some closed rational homology 3-sphere. This homeomorphism induces an isomorphism $h: \pi_{1}(N(\alpha)) \rightarrow$ $\pi_{1}\left(X\left(\alpha_{1}\right)\right) * \pi_{1}(W)$. Let $p_{1}: \pi_{1}\left(X\left(\alpha_{1}\right)\right) * \pi_{1}(W) \rightarrow \pi_{1}\left(X\left(\alpha_{1}\right)\right)$ be projection onto the first factor. Then $p_{1} \circ h$ sends $[L]$ to $n\left[L_{1}\right]$, for some $n \in \mathbb{Z}-\{0\}$. Hence, $\left[L_{1}\right]$ has finite order in $\pi_{1}\left(X\left(\alpha_{1}\right)\right)$.

Lemma A.20. Let $V$ be a compact connected orientable 3-manifold which is a rational homology solid torus. Suppose that $\partial V$ is a single torus $T$. Let $L$ be a knot in $V$ such that $V_{L}$ is a rational homology cobordism. Let $\mu$ be the meridional slope on $\partial \mathcal{N}(L)$ and let $r$ be another slope. Let $\mu_{1}$ and $r_{1}$ be the slopes on $T$ which are trivial in $H_{1}(V ; \mathbb{Q})$ and $H_{1}\left(V_{L}(r) ; \mathbb{Q}\right)$, respectively. Suppose that $T$ compresses in $V_{L}(r)$. Then there is a constant $K$ which depends only on $V$ (not on $L$ or $r$ ) such that $\Delta(r, \mu) \leq K \Delta\left(r_{1}, \mu_{1}\right)$. Furthermore, if $V\left(\alpha_{1}\right)$ is a homology 3-sphere for some slope $\alpha_{1}$ on $T$, then we may take $K$ to be 1 .

Proof. Throughout this proof, we shall arbitrarily assign an orientation to a slope on a torus, and so associate it with an element of the first homology of the torus. Let $\langle$,$\rangle be the intersection pairing on H_{1}(\partial \mathcal{N}(L) ; \mathbb{Q})$ or $H_{1}(T ; \mathbb{Q})$, as appropriate. It is a simple property of this pairing that if $x$ and $y$ are elements of $H_{1}(\partial \mathcal{N}(L) ; \mathbb{Q})$ which are homologous in $V_{L}$ to $x_{1}$ and $y_{1}$ respectively in $H_{1}(T ; \mathbb{Q})$, then $\langle x, y\rangle=\left\langle x_{1}, y_{1}\right\rangle$.

The boundary of the compression disc $D$ for $T$ in $V_{L}(r)$ has slope $r_{1}$. So, $D-$ $\operatorname{int}(\mathcal{N}(L))$ is a homology in $V_{L}$ between $r_{1}$ and $w r$ where $w$ is some integer. Note that $w$ is non-zero, since otherwise $r_{1}$ is homologically trivial in $V_{L}$, which is a contradiction.

Suppose that $\mu_{1} \in H_{1}(T ; \mathbb{Q})$ is homologous in $V_{L}$ to $z \mu \in H_{1}(\partial \mathcal{N}(L) ; \mathbb{Q})$, for $z \in \mathbb{Q} \backslash\{0\}$. We shall find a lower bound on $|z|$ which depends only on $V$. Given any slope $\alpha_{1}$ on $T$ which is not $\mu_{1}$, the manifold $V\left(\alpha_{1}\right)$ is a rational homology 3 -sphere. Thus $H_{1}\left(V\left(\alpha_{1}\right)\right)$ has finite order $K$, say. Pick such an $\alpha_{1}$ so that $K$ is minimal. Then $K$ depends only on $V$. Let $L_{1}$ be the core of the solid torus in $V\left(\alpha_{1}\right)$ which was glued onto $V$. The following is an excerpt from a Mayer-Vietoris exact sequence:

$$
0 \rightarrow H_{1}(T) \stackrel{i_{1 *} \oplus i_{2 *}}{\longrightarrow} H_{1}(V) \oplus H_{1}\left(\mathcal{N}\left(L_{1}\right)\right) \stackrel{i_{3 *}-i_{4 *}}{\longrightarrow} H_{1}\left(V\left(\alpha_{1}\right)\right) \rightarrow 0
$$

The maps $i_{j}$, with $j \in\{1,2,3,4\}$, are the relevant inclusion maps. Now,

$$
\left(i_{3 *}-i_{4 *}\right)\left(0, K\left[L_{1}\right]\right)=0 .
$$

Hence, there is an element $x_{1} \in H_{1}(T)$ such that $\left(i_{1 *} \oplus i_{2 *}\right)\left(x_{1}\right)=\left(0, K\left[L_{1}\right]\right)$. Then, $x_{1}=m_{1} \mu_{1}$, for some $m_{1} \in \mathbb{Z} \backslash\{0\}$. Note that $\left|m_{1}\right| \leq K$. Also, $x_{1}$ is homologous in $V_{L}$ to $m \mu$, for some $m \in \mathbb{Z} \backslash\{0\}$. Hence, $|z|=\left|m / m_{1}\right| \geq 1 / K$. Then, $\Delta\left(r_{1}, \mu_{1}\right)=\left|\left\langle r_{1}, \mu_{1}\right\rangle\right|=|\langle w r, z \mu\rangle| \geq|\langle r, \mu\rangle| / K=\Delta(r, \mu) / K$.

The following is a stronger version of Corollaries 2.4 and 2.7 of [1].

Theorem A.21. Let $N$ be a compact connected orientable irreducible 3-manifold with $\partial N$ a non-empty collection of tori. Let $S$ be a non-empty taut surface in $N$. Suppose that no non-empty collection of curves in $\partial S$ is homologically trivial in $\partial N$, and that no component of $S$ is closed and separating. Let $P$ be a component of $\partial N$ which is disjoint from $S$. Then, for all slopes $\alpha$ on $P$, except possibly one slope, the following statements hold in the manifold $N(\alpha)$ :

(i) $S$ is taut in $N(\alpha)$, and

(ii) each 2-sphere in $N(\alpha)$ bounds a rational homology ball, and 
(iii) the core of the surgery solid torus has infinite order in $\pi_{1}(N(\alpha))$.

Also, if the only tori in $N-S$ which are cobordant to $P$ by a rational homology cobordism are parallel to $P$, then

(iv) $N(\alpha)$ is irreducible.

Proof. If the only tori in $N-S$ which are cobordant to $P$ by a rational homology cobordism are parallel to $P$, then Theorem A.6 gives us the desired conclusion. Suppose therefore that $T$ is a torus properly embedded in $N-S$ which is cobordant to $P$ by a rational homology cobordism $H$. If $H_{2}(N, \partial S)$ is trivial, then each component of $S$ is closed and separating, which is a contradiction. Hence, by Lemma A.15 (with $F=\partial S$ ), $T$ is incompressible in $N$. Thus, by Haken's finiteness theorem [3, Lemma 13.2], we may assume that the only tori in $X=N-\operatorname{int}(H)$ which are cobordant to $T$ by a rational homology cobordism disjoint from $S$ are parallel to $T$.

Note that $S$ is taut in $X$. Let $\alpha_{1}$ be the slope on $T$ which is trivial in $H_{1}(H(\alpha) ; \mathbb{Q})$. If there is a 2-sphere in $N(\alpha)$ which does not bound a rational homology ball, then by Lemma A.11 (with $Q=N(\alpha)$ and $Y=H(\alpha)$ ), $X\left(\alpha_{1}\right)$ is reducible. If $S$ is not taut in $N(\alpha)$, then, by Lemma A.18, $S$ is not taut in $X\left(\alpha_{1}\right)$. If the core of the surgery solid torus in $N(\alpha)$ has finite order in $\pi_{1}(N(\alpha))$, then, by Lemma A.19, the core of the surgery solid torus in $X\left(\alpha_{1}\right)$ has finite order in $\pi_{1}\left(X\left(\alpha_{1}\right)\right)$. By Theorem A.6 (with $N, P$ and $\alpha$ of Theorem A.6 being $X, T$ and $\alpha_{1}$, respectively), there is at most one such $\alpha_{1}$, and hence there is at most one such $\alpha$.

There is also a sutured manifold version which is proved in exactly the same way, except that it uses Theorem A.9 instead of Theorem A.6.

Theorem A.22. Let $(N, \gamma)$ be a connected taut sutured manifold with $\partial N$ containing a toral component $P$. Suppose that $H_{2}(N, \partial N-P)$ is non-trivial. Then, for all slopes $\alpha$ on $P$, except possibly one slope, the following statements hold:

(i) each 2-sphere in $N(\alpha)$ bounds a rational homology ball, and

(ii) $R_{ \pm}(\gamma)-P$ is taut in $N(\alpha)$, and

(iii) the core of the surgery solid torus has infinite order in $\pi_{1}(N(\alpha))$.

Corollary A.23. Let $N$ be a compact connected orientable irreducible 3-manifold with $\partial N$ containing a toral component $P$. Then, for all slopes $\alpha$ on $P$, except possibly one slope, each 2-sphere in $N(\alpha)$ bounds a rational homology ball.

Proof. If $H_{2}(N, \partial N-P)$ is trivial and $\partial N=P$, then, for all slopes $\alpha$ on $P$ with one exception, $N(\alpha)$ is a rational homology 3 -sphere. The result then follows. If $H_{2}(N, \partial N-P)$ is trivial and $\partial N \neq P$, then by Lemma A.4 (d), $N$ is a rational homology cobordism between $P$ and $\partial N-P$. Hence, $N(\alpha)$ is a rational homology solid torus. An argument similar to the one used in Lemma A.11 then implies that each 2-sphere in $N(\alpha)$ bounds a rational homology 3-ball. If $H_{2}(N, \partial N-P)$ is non-trivial, then the result follows from Theorem 2.1 and Theorem A.22.

\section{REFERENCES}

[1] D. Gabai, Foliations and the topology of 3-manifolds, II, J. Differ. Geom. 26 (1987), 461-478. MR 89a:57014a

[2] C. Gordon and J. Luecke, Only integral Dehn surgeries yield reducible manifolds, Math. Proc. Camb. Phil. Soc. 102 (1987), 97-101. MR 89a:57003

[3] J. Hempel, 3-Manifolds, Ann. of Math. Studies, No. 86, Princeton Univ. Press, Princeton, N. J., 1976. MR 54:3702 
[4] W. Jaco, Lectures on Three-Manifold Topology, Regional Conference Series in Mathematics, No. 43, Providence 1980, A. M. S. MR 81k:57009

[5] R. Kirby, A calculus for framed links in $S^{3}$, Inv. Math. 45 (1978), 36-56. MR 57:7605

[6] M. Lackenby, Surfaces, surgery and unknotting operations, To appear in Math. Ann.

[7] M. Lackenby, Upper bounds in theory of unknotting operations, To appear in Topology.

[8] W. B. R. Lickorish, A representation of orientable combinatorial 3-manifolds, Ann. of Math. 76 (1962), 531-540. MR 27:1929

[9] M. Scharlemann, Sutured manifolds and generalized Thurston norms, J. Differ. Geom. 29 (1989), 557-614. MR 90e:57021

[10] M. Scharlemann, Producing reducible 3-manifolds by surgery on a knot, Topology 29 (1990), 481-500. MR 91i:57003

[11] M. Scharlemann, Unlinking via simultaneous crossing changes, Trans. Amer. Math. Soc. 336 (1993), 855-868. MR 93k:57021

[12] P. Scott, The geometries of 3-manifolds, Bull. London Math. Soc. 15 (1983), 401-487. MR 84m:57009

Department of Pure Mathematics and Mathematical Statistics, University of CamBridge, 16 Mill Lane, Cambridge, CB2 1SB, England

E-mail address: ml128@pmms.cam.ac.uk 